\title{
Strukturen und Strategien in der epigraphischen Kommunikation des kommunalen Italiens
}

Diesem Aufsatz möchte ich drei Prämissen bzw. Präzisierungen vorweg schicken. Die erste betrifft mein Untersuchungsvorhaben, das sich als allzu gewagt und ehrgeizig erweisen könnte. Ich bin mir der Themenweite und der ihr entgegenstehenden Umfangsbegrenzungen allerdings sehr genau bewusst. Auch kenne ich das Wagnis, Fragen und Materialien summarisch $\mathrm{zu}$ behandeln, die auch andere Autor*innen dieses Sammelbandes untersucht haben. Daher nehme ich mir vor, überflüssige Ausführungen zu vermeiden und mich auf die Strukturen zu beschränken, die in der Geometrie als ,ausgezeichnete Elemente‘, also als Konstanten bezeichnet werden. Damit meine ich jene Elemente, die mir die Grundstrukturen des Systems der epigraphischen Kommunikation im kommunalen Italien zu sein scheinen. Ich bin nämlich der Ansicht, dass die Gesamtheit der Inschriften aus der Zeit der Kommunen, die gut identifizierbare Sender und Empfänger sowie charakteristische Kommunikationsstrategien und privilegierte Ausstellungsorte besitzen, berechtigterweise als ein System bezeichnet werden kann.

Meine zweite Präzisierung greift einige Ideen auf, die ich bereits vor vielen Jahren über das enge Verhältnis zwischen der Welt der italienischen Kommunen und den „scritture esposte“, d. h. den Inschriften entwickelt habe. ${ }^{1}$ Der von Armando Petrucci geprägte Ausdruck „scritture esposte“ hat im Rahmen der mittelalterlichen Studien zur Inschriftenkultur große Bedeutung erlangt. Ohne konzeptuelle Unterschiede vorzunehmen, werde ich ihn deckungsgleich mit dem Begriff ,Inschriften“ verwenden. ${ }^{2}$ Hierzu sei am Rande angemerkt, dass sich in einer Geschichtsschreibung, die die Tendenz besitzt, Sachverhalte zu sehr zu vereinfachen, äußerst unterschiedliche Quellen vereinen. Die zweifellos ausgeprägte Doppelkonnotation der Quellenmaterialität und -ausstellung bildet ein Element der Einheitlichkeit - besser noch: eine

1 Vgl. Giovè 1994.

2 Die Definition der „scritture esposte“, der „öffentlich ausgestellten Schriften“ verweist auf jedwede Art von Schrift, die erzeugt wird, um das Lesen eines schriftlichen, mitunter weiter entfernt liegenden Textes auf einer ausgestellten Oberfläche durch mehrere Leser zu ermöglichen. Sie ist nachzulesen bei Petrucci 1985, 88. Dieser und ein weiterer Aufsatz von Petrucci aus dem Jahr 1986 sind Meilensteine in der Erforschung mittelalterlicher Inschriftenkultur. Sie sind aufgrund der Perspektiven, die sie eröffnen, sowie der terminologischen Vorschläge, die sie machen, unentbehrlich.

Mein herzlicher Dank gilt den Organisatoren für die Einladung zur Tagung in Rom sowie dafür, dass sie mir auf diese Weise ermöglicht haben, die Flamme einer alten Liebe wieder anzufachen, die niemals erlöscht.

Ә Open Access. () 2019 Nicoletta Giovè Marchioli, publiziert von De Gruyter. (œ) Bł-Nc-ND Dieses Werk ist lizenziert unter der Creative Commons Attribution-NonCommercial-NoDerivatives 4.0 Lizenz. 
Verpflichtung zu dieser, die gegebenenfalls die Unterschiede zwischen den verschiedenen Inschriftentypen nivelliert und verschleiert. Wenn ich allerdings von kommunaler Inschriftenkultur spreche, so meine ich stricto sensu nicht allein jene, die eine gegebene politische Realität wiederspiegelt, die ihrerseits im Rahmen eines spezifischen gesellschaftlichen Organisationssystems sowie bestimmter sozialer Beziehungen entstanden ist. Vielmehr verweise ich ebenso, lato sensu, auf jene politische Realität, die sich zwischen dem 11. und 14. Jahrhundert im zentralnördlichen Italien entwickelte.

Die letzte einleitende Überlegung betrifft den Optimismus, und zwar jenen Optimismus, der mir vor fünfundzwanzig Jahren noch fehlte, als ich begann, mich mit mittelalterlichen Inschriften $\mathrm{zu}$ beschäftigen. In dieser Zeit erschien mir die Forschungslage desolat und düster. Dass ich mich dem Thema jetzt so zuversichtlich widmen kann, ist dem Reichtum an Initiativen geschuldet, die sowohl in Italien als auch in einem größeren europäischen Kontext ergriffen wurden. Dieser Sammelband ist ein weiteres Ergebnis dieses Wissensdranges.

\section{Einige Grundbegriffe}

Die allgemeine Funktion von Inschriften wird bei Augustinus in einer Passage der Sermones de sanctis zum Totengedenken und zur Rolle der Epitaphien deutlich. In dieser dem Märtyrer Stephan gewidmeten Predigt heißt es, diese erlaubten, dass qui vult, legat und quando vult, legat, weil sie gerade hierzu dienten: ut omnes legant. ${ }^{3}$ Hingewiesen wird also auf eine der wesentlichen Funktionen der „scritture esposte“, und zwar die Zurschaustellung einer Botschaft. Darüber hinaus erfüllten die Inschriften allerdings weitere bedeutende Funktionen, nämlich die zeitliche Festlegung einer Botschaft, die Möglichkeit ihrer Wiederholung und ihrer emphatischen Verstärkung. Dies trifft umso mehr zu, wenn die Botschaft mittels einer Synergie von Schrift und Figur, Text und Bild organisiert und verkündet wurde. Wenn ich hier diese Wendung gebrauche, die beinahe wie eine Art Hendiadyoin klingt, dann, weil mit Synergie zum Ausdruck gebracht werden soll, dass das Wort von einer ikonographischen Version des damit Bezeichneten begleitet wird oder - im Gegensatz dazu - eine bildliche Darstellung mit einem Begriff einhergeht, der sie erklärt und ergänzt.

Legen wir an dieser Stelle einige Punkte fest, von denen wir im Folgenden ausgehen wollen: Die Bedeutung des ideologischen Wertes, der Organisation sowie der spezifischen Gestalt eines epigraphischen Denkmals kann nur dann vollständig verstanden werden, wenn wir diese im Rahmen der Spielformen betrachten, welche die „scritture esposte“ mit der Zeit entwickelten. Ohne den (vergeblichen) Versuch unternehmen zu wollen, hier die Geschichte der latinischen Inschriftenkultur darzulegen

3 Augustinus von Hippo, Sermo 319, De Stephano martyre VI, 7. 
und zusammenzufassen, sei nur daran erinnert, dass die römische Gesellschaft auf schriftlicher Kommunikation basierte und geradezu vehement eine aktive Verbreitung des schriftlichen Wortes betrieb, wobei sie aber keineswegs nur auf traditionelle Textträger beschränkt blieb. Die klassische Welt glich folglich einem Universum, in dem das öffentlich ausgestellte Wort herrschte - mit dem Zweck, eine möglichst große Anzahl an Adressaten zu erreichen, denen wiederum eine Botschaft mit dem Anspruch von höchster Publizität vermittelt wurde. Das Christentum reihte sich in die klassische Tradition ein und begann, eine ausgiebige und bewusste Verwendung des öffentlich ausgestellten Wortes zu fördern. Im Frühmittelalter verschwand diese Praxis dann beinahe vollkommen, sodass die Ausstellung schriftlicher Botschaften im freien Stadtraum praktisch gar nicht mehr vorkam und sich zunehmend auf den Innenbereich religiöser Gebäude beschränkte. ${ }^{4}$ Das Spätmittelalter hingegen bedeutete für das Inschriftenwesen eine regelrechte Revolution, denn es erlangte insbesondere in Italien, wo es zunehmend an Bedeutung gewann, erneut einen starken ideologischen und propagandistischen Wert, wie nicht zuletzt sein intensives Wiederaufleben innerhalb der Kommunen belegt. Diese traten ihrerseits als Begleiterscheinung einer renovatio-urbium-Bewegung auf, worunter sowohl ein urbanistischer Ausbau der in kontinuierlicher Bauexpansion befindlichen Städte als auch ein wirtschaftlicher und kultureller Aufschwung zu verstehen sind. ${ }^{5}$

Folglich beobachten wir innerhalb der „scritture esposte“ des Mittelalters die auffällige Zäsur einer Zeit davor und einer Zeit danach, denn unbestreitbar erneuerten die aufstrebenden Führungsgruppen der italienischen Kommunalstädte, die vom Wert der schriftlichen Kommunikation überzeugt waren, den Gebrauch von Inschriften. Sie nutzten gekonnt und gezielt die besondere Sprache der epigraphischen Schrift und vertrauten ihr die vielfältigsten Inhalte an. Entsprechend erfuhr das kommunale Italien eine immer intensivere Verwendung des öffentlich ausgestellten Wortes. Dieses entwickelte sich zu einem bedeutenden Kommunikationsmittel und nahm seinerseits eine wichtige Stellung innerhalb der politischen Ereignisgeschichte ein, während es andererseits neue Bereiche durchdrang. Somit wurde der städtische Raum des italienischen Mittelalters auch bzw. erneut eine Bühne für öffentliche Schrift und gleichzeitig selbst ein graphisch geprägter Raum, der sich selbst in kontinuierlicher Expansion befand.

Ergänzend sei daran erinnert, dass epigraphische Denkmäler unter anderem ein ausgezeichnetes Instrument zur Erforschung unterschiedlicher Modi der Selbstdarstellung sind, wobei wir uns stets ihr Grundmodell vor Augen halten sollten, wonach Text, Bild und Kontext integriert zusammenwirken. So ist es unerlässlich, zunächst das Verhältnis zwischen Schrift und Monument zu analysieren, also die materielle

4 Vgl. hierzu auch die Beiträge von Marialuisa Bottazzi und Katharina Bolle in diesem Band.

5 Zur Expansion urbaner Zentren im Italien der kommunalen Zeit siehe beispielhaft den Band $L a$ costruzione della città comunale italiana 2009. 
wie ideologische Verbindung, die die Schrift und den Träger, auf dem sie angebracht ist, zusammenhält. Besonders innerhalb der städtisch-kommunalen Inschriftenkultur konkretisierte sich diese Verflechtung in Artefakten mit einer großen Kommunikations- und Suggestionskraft, die darüber hinaus eine unmittelbare Wirkungsmacht entfalteten.

\section{Vergangenheit, Gegenwart und Zukunft}

Die Grabepigraphik möchte ich in diesem Aufsatz absichtlich ausklammern, obgleich sie meinem Thema nur scheinbar fernliegt, da in einigen Städten die Wahl eines spezifischen Umfelds für die Errichtung monumentaler Begräbnisstätten eine konkrete Bedeutung hatte. Diese Positionierung kann im weitesten Sinne auch aus einer städtischen oder vielmehr politischen Perspektive heraus relevant sein, wie sie von den - wenngleich nicht häufigen - Texten eingenommen wird, die die „scritture esposte“ begleiteten. Dabei denke ich beispielsweise an Bologna und die Arche dei Glossatori (Abb. 1), also an die monumentalen Gräber von einigen der wichtigsten Professoren des römischen Rechts am Studium von Bologna im Spätmittelalter. Die bis heute erhaltenen fünf Grabmäler, die alle auf die zweite Hälfte des 13. Jahrhunderts zurückgehen, ragen auf den Plätzen empor, die den Basiliken von S. Francesco und S. Domenico gegenüberliegen. Derart prominent im Stadtbild positioniert, besetzten sie wesentliche Knotenpunkte des Stadtraums in Verbindung mit großen religiösen Gebäuden, die ihrerseits die wirksame Präsenz der Bettelorden in der Stadt zum Ausdruck brachten und zugleich verstärkten. Auf diese Weise wurden die städtischen Berühmtheiten mit den städtischen Kirchen vereint. ${ }^{6}$

In den sich ständig wandelnden Kontexten der italienischen Stadtkommunen des Spätmittelalters - beginnend mit den chronologisch ältesten und gleichsam experimentelleren - entwickelte sich die inschriftliche Sprache zum bevorzugten Kommunikationsmedium. Ich beziehe mich hier auf die „scritture esposte“, die von einer öffentlich-laikalen Autorität geschaffen wurden - also auf eine gewissermaßen ,zivile‘ Inschriftenkultur, von der das Echo antik-römischer Praktiken widerhallt. Die von Inschriften getragene Kommunikation schließt sowohl das Gewöhnliche als auch das Außergewöhnliche ein. Gleichzeitig liefert sie Details über die Regelung des städtischen Lebens, erinnert aber auch an einmalige und deshalb erinnerungswürdige

6 Diese einen äußerst originellen Monumentaltypus bildenden Gräber weisen eine gemeinsame Struktur auf. Sie bestehen aus einer Ädikula, die von einer Pyramide überragt wird und sich auf Marmorsäulchen stützt. Die Säulchen sind ihrerseits auf kleinen Pfeilern oder einem Sockel aufgestellt. In ihrem Inneren befindet sich ein Marmorsarkophag. Das Gedenken an verstorbene Berühmtheiten wurde allerdings nur selten dem geschriebenen Wort anvertraut: Vgl. Grandi 1978/79 und (in Erweiterung der Überlegungen) Grandi 1982. 


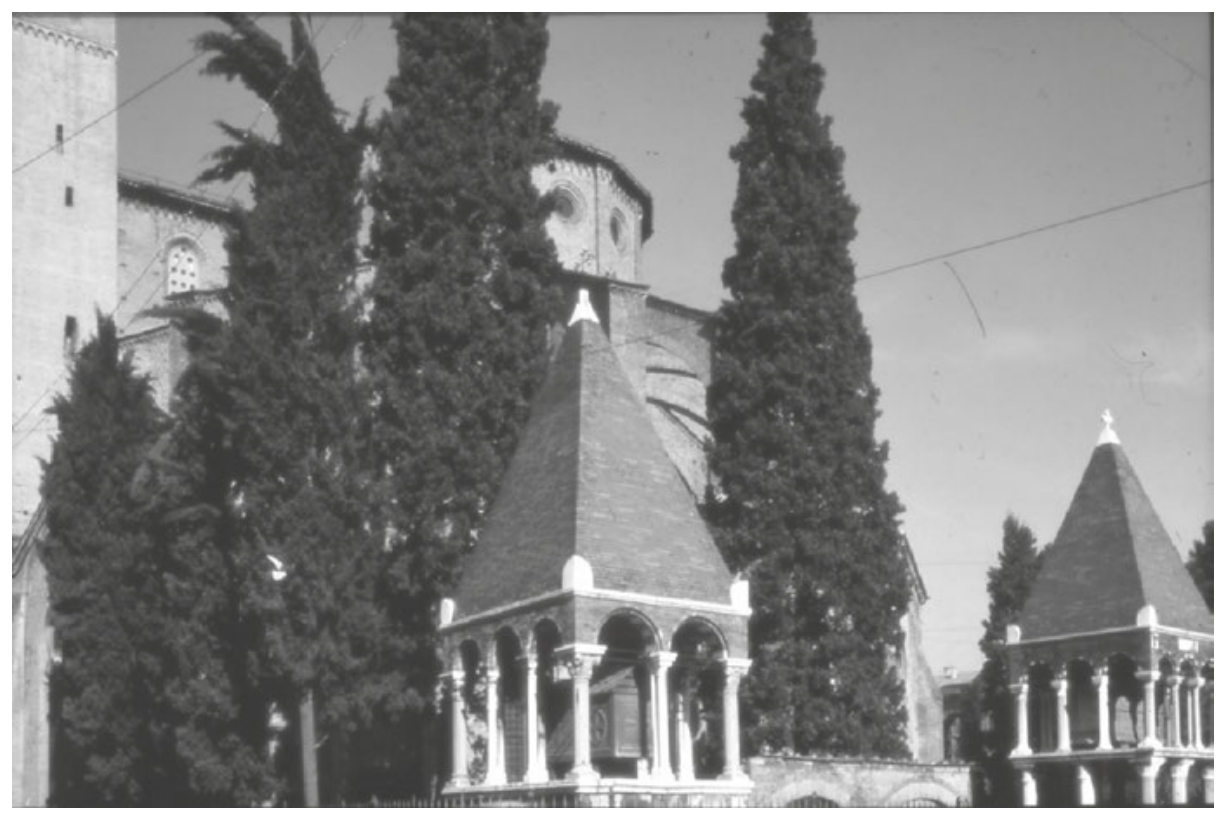

Abb. 1: Arche dei Glossatori in Bologna: (C) wikimedia commons.

Ereignisse, womit sie einen Bogen von der reinen Information über das Gedenken bis hin zur Verherrlichung spannt. Dieser Bandbreite an Funktionen entspricht ein Spektrum unterschiedlichster Realisierungsmöglichkeiten, die von den bescheidensten und auf das Essentielle beschränkten Ausfertigungen bis hin zu den elaborierteren und monumentalen Exemplaren reichen. Daher ist nicht immer ganz leicht festzustellen, ob - mehr oder weniger bewusst - das Aktuelle oder das Vergangene fokussiert wird, ob die Emphase auf der Gegenwart oder der Zukunft liegt und demnach, worin die Wechselwirkung zwischen der absichtsvollen Herstellung eines inschriftichen Denkmals und der Rezeption durch den Betrachter desselben besteht. Wir können postulieren, dass alle Inschriften naturaliter in einem irgendwie gearteten Verhältnis zur Gegenwart stehen, jedoch auch mit einer Perspektive auf die Zukunft gedacht werden können. Vermutlich bestimmte die Bedeutung der Nachricht ideell den bevorzugten Adressaten, womöglich unter Berücksichtigung dessen jeweiliger Lesefähigkeit. An dieser Stelle sei daran erinnert, dass wir uns innerhalb eines „langen Spätmittelalters“ bewegen. Diese chronologische Ausdehnung ermöglicht es, besondere und originelle Aspekte einiger Entscheidungen zu differenzieren. 


\section{Brunnen als epigraphische Monumente}

Die hierfür stehenden Beispiele sind zahlreich. Als Einstieg möchte ich eines der auffälligsten nennen: die Fontana Maggiore von Perugia. Dieses bemerkenswerte Kunstwerk liegt mir und Attilio Bartoli Langeli, mit dem ich es gemeinsam studiert habe (Abb. 2), ${ }^{7}$ sehr am Herzen. Die Komplexität und Originalität des Brunnens liegt maßgeblich in den eingravierten Schriftzügen begründet, die ihn zu einem nicht nur von Figuren, sondern auch von Wörtern geprägten Denkmal machen. Somit vermittelt er seine Botschaften nicht nur symbolisch, sondern auch graphisch.

Bevor wir darauf zu sprechen kommen, bedarf es aber noch einiger einleitender Worte: Die Brunnen - und mithin das Wasser - waren nicht nur eines der wichtigsten öffentlichen Güter der italienischen Kommunen, sondern darüber hinaus auch ein beliebtes Element, zur Verschönerung der Stadt beizutragen. ${ }^{8}$ Folglich verbanden sie utilitas mit decus, also Nutzen mit Zierde. Scharfsinnig wird diesem Umstand in einem bedeutungsvollen Aufsatz von Attilio Bartoli Langeli und Sonia Merli Rechnung getragen, der sich mit Brunnen und ihrer Bedeutung auf dem Feld der öffentlichen Dienstleistung im urbanen Bereich beschäftigt. ${ }^{9}$ Auf vielen Brunnen dieser Zeit finden sich auch graphische Ausstattungselemente, die oftmals mit den Unterschriften der Künstler beginnen, die sie geschaffen haben. Nun sind mit Inschriften versehene Bauten der Wasserversorgung wie Brunnen oder auch Aquädukte kein auf Perugia beschränktes Phänomen; auch anderswo brachte man ihnen große Wertschätzung entgegen. Um die Bedeutung zu unterstreichen, die derartigen Monumenten zugeschrieben wurde, ist eine ideelle Rückbesinnung auf Rom und auf zwei zeitlich durchaus weit auseinander liegende Zeitspannen seiner Geschichte hilfreich, nämlich die klassische Antike einerseits und das 16. und 17. Jahrhundert andererseits. Auch und vor allem in der Zeit der Kommunen waren diese Werke mit „scritture esposte“ versehen - und dies durchaus zahlreich, obwohl die Inschriften weniger dazu bestimmt waren, gelesen als vielmehr betrachtet zu werden. Dies legt jedenfalls die mitunter stark eingeschränkte Lesbarkeit dieser Texte nahe. Man denke nur an Viterbo und an seine zahlreichen schönen Brunnen, die eingraviert die Erinnerung derjenigen Personen tragen, die an ihrem Bau beteiligt waren. Dabei handelt es sich sowohl um die Handwerker, die die Brunnen konkret schufen, als auch um die Staatsmänner und die Stadtregierenden, die sie in Auftrag gaben und die man ihrerseits für ihre so demonstrierte benignitas würdigte. Der bekannteste Brunnen von Viterbo, die aus Peperin gebaute Fontana del Sepale, später auch Fontana Grande genannt, geht in seiner ursprünglichen Struktur auf das Ende des 12. Jahrhunderts zurück. Er ist

7 Vgl. Bartoli Langeli/Giovè Marchioli 1996, vertieft von Bartoli Langeli/Zurli 1996.

8 Verwiesen sei hier zumindest auf die Vorschläge von Gouédo-Thomas 1992, Schulze 1994 und zuletzt Schmid 1998 sowie auf das jüngere Verzeichnis von Visentin 2016.

9 Vgl. Bartoli Langeli/Merli 2013. 


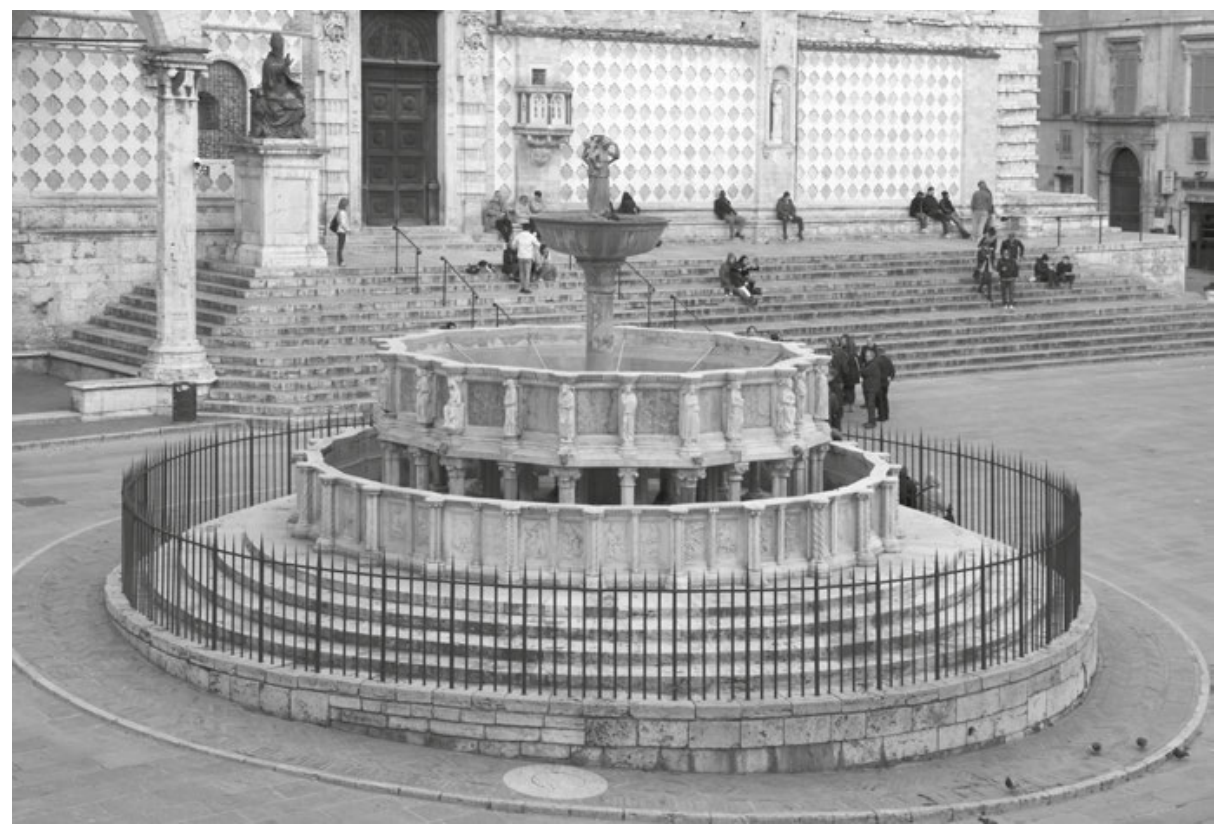

Abb. 2: Fontana Maggiore von Perugia: (C) wikimedia commons.

durch ein großes Vierpassbecken charakterisiert und trägt entlang der Ränder aller vier Brunnenbecken eine große Inschrift aus dem Jahr 1279 (Abb. 3). ${ }^{10}$

Ungewöhnlicherweise wird in der Inschrift nicht an den Erbauer des Brunnens erinnert - obgleich mit dem römischen Podestà Orso Orsini und dem Capitano del Popolo Arturo di Pietro di Raniero Geizone städtische Autoritäten durchaus genannt werden. In einer zweiten Inschrift, die um den achteckigen Brunnensockel verläuft, wird außerdem der Name des Brunnenrestaurators Valeriano erwähnt. Von dieser sind heute aber nur die ersten zwei leoninischen Hexameter lesbar. Dieses für uns befremdliche Verschweigen des Erbauers wird verständlicher, wenn wir uns klarmachen, dass die mit einem Brunnen verbundene Erinnerung (hier diejenige an seinen Schöpfer) häufig eher suggeriert und in den alphabetischen Zeichen gleichsam ideographisch zusammengefasst wurde (mir sei das Oxymoron verziehen) - auch wenn die Anordnung dieser Zeichen unserer Vorstellung von Lesbarkeit entgegensteht.

Die Inschriften aus Volterra, die die Einrichtung der Fonte di Docciola von 1245 (Abb. 4) und der Fonte di S. Felice von 1319 (Abb. 5) kommemorieren, scheinen in diesem Sinne einen Kompromiss dazustellen. ${ }^{11}$

Die erste Inschrift ist auf einer rechteckigen Platte eingraviert, die mehr als einen Meter lang, aber nur um die zwanzig Zentimeter hoch ist und in das Mauerwerk des

10 Vgl. Carosi 1986, 86-89 Nr. 30.

11 Vgl. Augenti/Munzi 1997, 59-61 Nr. 8 und 76-78 Nr. 17. 


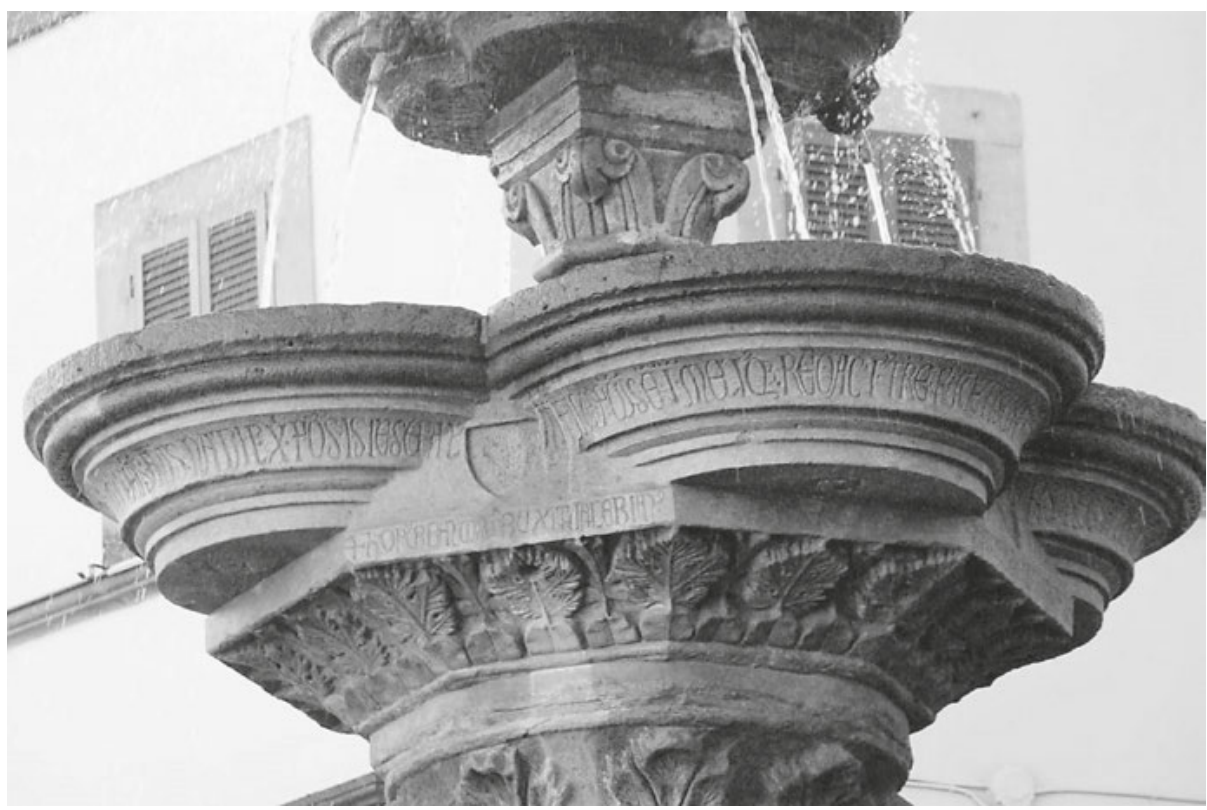

Abb. 3: Inschrift der Fontana del Sepale von Viterbo: Foto @ Carlo Tedeschi.

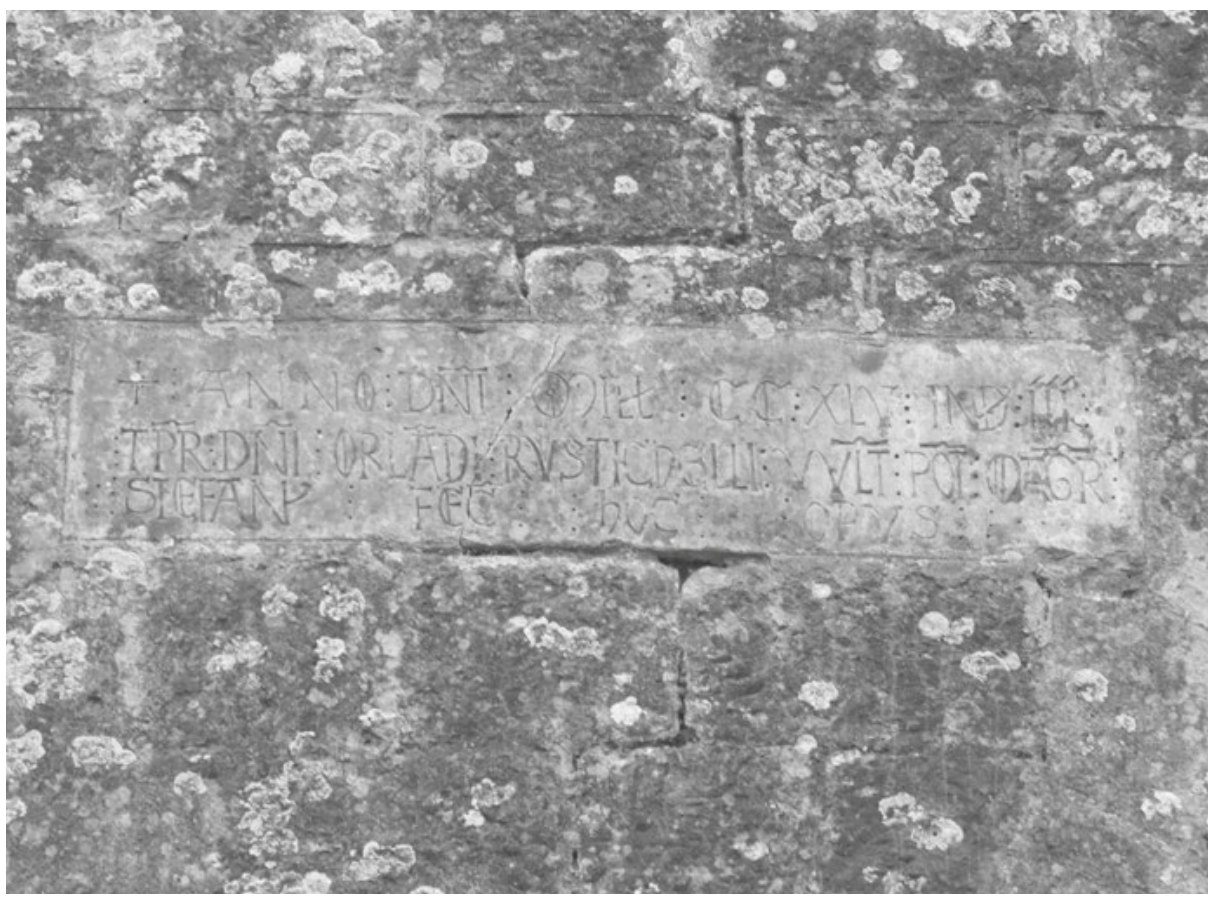

Abb. 4: Inschrift der Fonte di Docciola von Volterra: @ wikimedia commons. 


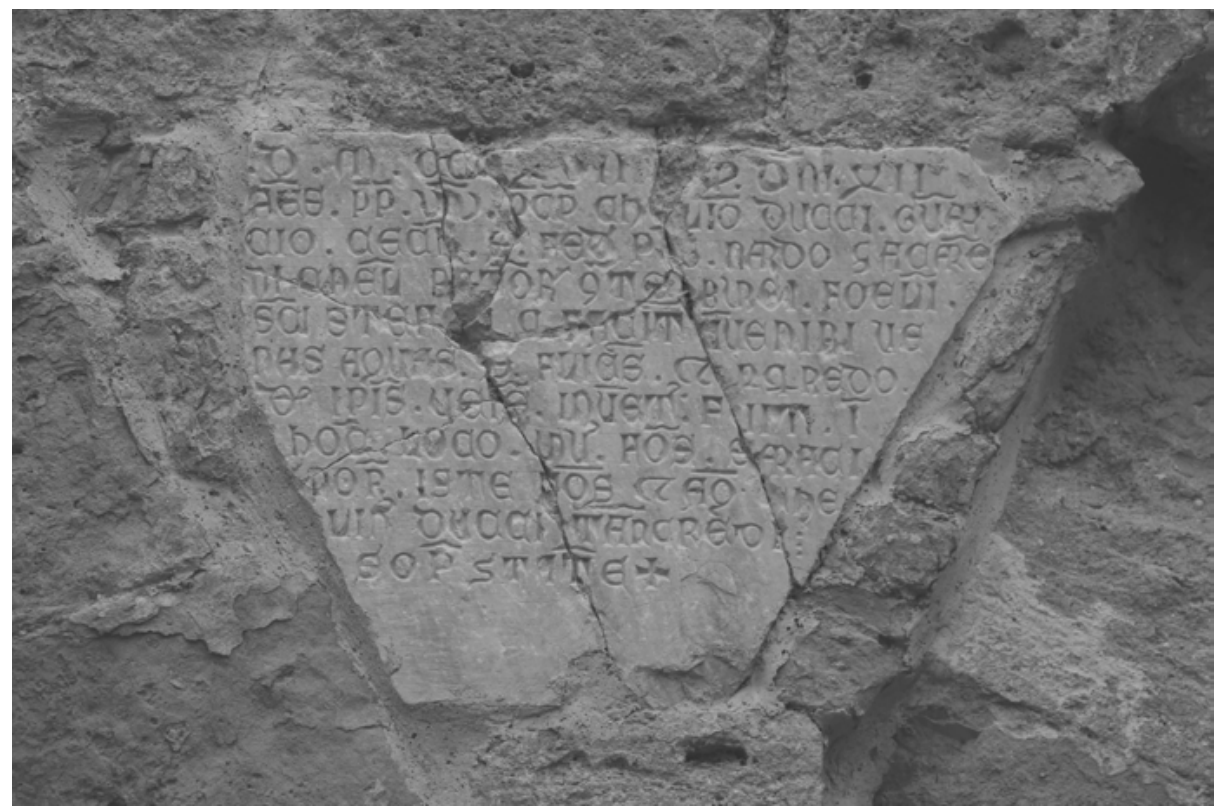

Abb. 5: Inschrift der Fonte di S. Felice von Volterra: (C wikimedia commons.

Brunnenvorbaus eingefügt wurde. Die zweite steht auf einer trapezförmigen Platte in dem Pfeiler, der die zwei Vorbauarkaden stützt. Beide Inschriften sind vergleichsweise gut lesbar, obgleich die erste durch die Stütze aus Tuffstein zum Teil überdeckt wird, und die zweite durch ihre geringe Größe von ungefähr einem halben Meter Schwierigkeiten bereitet. Während die erste regelrecht lakonisch anmutet und bloß die Namen des verantwortlich zeichnenden Podestà Orlando Rustichelli und des ausführenden magister Stefano nennt, zeichnet sich die zweite durch eine geradezu gegensätzliche Mitteilsamkeit aus. Ihr Text verfolgt den Weg der Kommunalverwaltung bei der Durchführung der Brunnenerrichtung zurück: Demnach waren es die zwölf defensores der Stadt, die die balitores der Viertel von S. Felice und S. Stefano, also Amtspersonen, die für die Wartung der Brücken und Brunnen verantwortlich waren, mit der Suche nach einer geeigneten Quelle beauftragten. Einer von ihnen wurde außerdem damit betraut, die Arbeit zu beaufsichtigen.

Besondere Erwähnung verdient auch die Erinnerungsstiftung des Aquäduktbaus von Pietrare in Viterbo. Die entsprechende Inschrift wurde im Jahr 1268 nicht ganz ohne Schwierigkeiten in die Felsspitze gehauen, die den Anfang des Aquädukts selbst markierte. Die Felsspitze wurde in jüngster Zeit zerstört und die Inschrift zerteilt in ein Museum überführt. Den zentralen Kern der Inschrift bildet die Erwähnung desjenigen, der das Werk realisieren wollte: Visconte, von dem außerdem gesagt wird, er sei der stirps Raneri Gacti angehörig, also Mitglied einer der bedeutendsten Familien 
der Stadt. Darüber hinaus sei er prudens und vi Verbi capitaneus, folglich ein Capitano del Popolo nach dem Willen Gottes. ${ }^{12}$

Ich kehre an dieser Stelle noch einmal zum Brunnen von Perugia zurück, um an seinem Beispiel die Vielzahl der Kommunikationsebenen $\mathrm{zu}$ verdeutlichen, welche Inschriften anstreben und verwirklichen konnten. Auch wenn in der Fontana Maggiore das bildliche Element das graphische zu überragen scheint, so verdienen beide Elemente doch die gleiche Aufmerksamkeit. Entlang des Außenrandes des Bronzebeckens erinnert eine Inschrift daran, wie das Werk tempore Gerardini de Buskettis potestatis et Anselmi de Alçate capitanei populi realisiert wurde. Sie erwähnt ferner, dass dessen magistri der Benediktiner Bevegnate und Bonasegna gewesen seien, während Rubeus als eigentlicher Schöpfer des Brunnens genannt wird. Rund um die Zentralsäule, die das Becken stützt, findet sich ein weiterer Schriftzug, der auf das Jahr 1278, tempore domini Mathei de Corigia et domini Ermanni de Saxoferrato, datiert ist. So spärlich der Informationsgehalt dieser Inschriften auch sein mag, sie beeindrucken dennoch, da es sich um besonders schön gestaltete Auszeichnungsschriften handelt. Zwar konnten sie aufgrund der Anbringung in einiger Höhe kaum vom Boden aus gelesen werden; sie optisch ansprechend $\mathrm{zu}$ gestalten wurde aber offensichtlich dennoch als wichtig empfunden.

Im unteren Becken erstreckt sich eine Inschrift über die 25 kleinen Marmoreinfassungen, die auf der oberen Seite die Fliesenpaare begrenzen, welche ihrerseits die Flachreliefs tragen. Im oberen Becken findet sich eine Inschrift, die auf den 24 Einfassungen verläuft, die sich unter den roten Marmorplatten befinden. In jeder dieser Einfassung ist ein Vers eingraviert. Schließlich wurden Inschriften auch oberhalb des zweiten Beckens angebracht, die auf den oberen Einfassungen der Platten lokalisiert sind. Diese beziehen sich auf die Figuren, die auf den angrenzenden Statuetten dargestellt sind. Jede Einfassung ist mit der zweiten Hälfte einer dargestellten Schriftrolle versehen, die mit dem linken Bild verbunden ist sowie mit der ersten Hälfte einer Schriftrolle, die mit dem rechten Bild verbunden ist. Somit handelt es sich um Inschriften, die - wie einem Dominospiel gleich - äußerst originell aneinander gekettet sind. Hieraus ergibt sich ein ununterbrochener Schriftzug, der perfekt an die sequenzielle Anordnung der 24 Statuen anschließt. Diese sind ihrerseits nach einem Schema aufgestellt, das sich am Windrosenmodell ausrichtet. So werden Symmetrieund Spiegelverhältnisse in einer kreisförmigen Sequenz fortwährender Querverweise bestimmt.

Die eigentliche Ausführung des Brunnens und seiner Inschriften wird Nicola und Giovanni Pisano zugewiesen, also zwei herausragenden und schaffensfreudigen Künstlern, die als die einflussreichsten Vertreter der gotischen Bildhauerei im Italien des 13. Jahrhunderts gelten können. Der Schriftgebrauch bei religiösen und zivilen

12 Vgl. Carosi 1986, 66-69 Nr. 22. Neben der Inschrift war das Familienwappen eingraviert, das in späterer Zeit allerdings abgeschliffen wurde. 
Denkmälern, der immer an die spezifischen Besonderheiten ihrer Gemeinde und deren Geschichte gebunden war, wuchs mit der Entwicklung der politischen Ordnung in den italienischen Kommunen. Er diente nicht zuletzt dazu, die Geschicklichkeit des Künstlers und dessen entscheidenden Beitrag zum Werk ins Gedächtnis der Stadt einzuprägen. Insbesondere die Familie Pisano begnügte sich wie viele andere nicht damit, zahlreiche ihrer Skulpturen bloß zu „unterschreiben“, sondern entwickelte eine systematische Verwendung der „scritture esposte“. Sich den Brunnen - seines Zeichens Stadtdenkmal und Ausdruck eines sehr ausgeprägten Bürgerbewusstseins - als Resultat eines geteilten Kreativprozesses vorzustellen, in dem auf der einen Seite die Autoren und auf der anderen die Auftraggeber stehen, geht allerdings an der Realität vorbei: Mit Sicherheit waren die Auftraggeber des Brunnens, also die Mitglieder Stadtregierung, auch direkt an der Textkonzipierung beteiligt.

Die Brunneninschriften sind vorwiegend von lehrhaftem Charakter: Indem sie einen Gegenpol zu den Bildern darstellen, veranschaulichen und erklären sie diese. Sie weisen eine kommunikative Funktion auf, die den Text unterstützt und wiederholt. Sie bieten jedoch zudem eine Nachricht in Buchstabenform, die unabhängig von ihrer Lesbarkeit einen eigenen Sinn besitzt. Dies ist ein Paradox, das nicht nur auf die Inschriften des Brunnens von Perugia zutrifft, sondern auch auf zahlreiche andere Inschriften aus kommunaler Zeit, deren spezifische Materialität eine unmittelbare Verbindung zwischen Sicht- und Lesbarkeit zu verhindern scheint. Das Problem, den Grad an Lesbarkeit zu bestimmen, erweist sich in diesem wie in vielen anderen Fällen als entscheidend, denn es zwingt uns, über die jeweiligen Gegebenheiten nachzudenken, die die Lesbarkeit der Inschrift beeinträchtigen. Davon sind unter anderem sehr bemerkenswerte Inschriften betroffen: Einerseits befinden sie sich an Stellen, die für den Blick kaum, wenn nicht unmöglich zu erreichen sind; andererseits weisen sie ein mitunter stark ornamentiertes Schriftbild auf, das dem Leser eine gewisse Konzentration abfordert.

Neben und über diese selbstverständlichere, gewissermaßen „alphabetische“ Funktion des sprachlichen Verständnisses hinaus erfüllten Inschriften eine hyperkommunikative Funktion, indem sie einen absoluten ikonischen Wert einnahmen. Jede Inschrift war und ist selbst Bild und Symbol. Die wirkungsmächtige Verbindung von Schrift und Bild stellt sich in vielen mittelalterlichen Kunstwerken, insbesondere den kommunalen, als ein grundlegendes Element heraus. Ihren stärksten Ausdruck findet sie im Denkmal von Perugia, bei dem Text und Bild in einem Verhältnis der vollkommenen und gegenseitigen Ergänzung stehen. Im Zusammenspiel entwickeln sie eine starke und gefestigte Interaktion und bilden ein sprachliches und visuelles Kommunikationssystem. Text und Bild konvergieren darin, dieselbe Information $\mathrm{zu}$ liefern, sie verbinden sich miteinander und verstärken sich wechselseitig. Somit setzen sie eine integrierte Kommunikationsbeziehung um, die die aktive und bewusste Anwesenheit eines Beobachters und Lesers voraussetzt. Entsprechend können sie eine Funktion erfüllen, die rein narrativ oder aber auch dialogisch sein kann. 


\section{Inschriften auf Toren und Mauern}

Im kommunalen Italien waren Inschriften, die an die Erbauung, Erweiterung oder Restaurierung mehr oder weniger monumentaler öffentlicher Werke erinnern, weit verbreitet. Als Beispiele für solche Bauwerke sind neben Brunnen auch Amtsgebäude städtischer Autoritäten zu nennen, vor allem aber die Tore und Mauern einer Stadt. Die mit ihnen verbundenen Inschriften konnten textlich durchaus unterschiedlich gestaltet sein. Während manche Angaben, wie etwa die Datierung, fast immer vorhanden waren, kamen bei anderen Inschriften weitere Informationen hinzu. So wurden die Inschriften beispielsweise um die Namen der Amtspersonen ergänzt, die eine Initiative gefördert hatten, ebenso wie um diejenigen der Künstler, die das Werk konzipiert und konkret realisiert hatten.

Die „scritture esposte“ derartiger Kontexte zelebrierten selbstverständlich nicht nur Gegenständliches, sondern auch Handlungen, indem sie neben der Bautätigkeit auch kriegerische Taten und politische Leistungen erwähnten - und beides häufig miteinander in Beziehung setzten. Die inschriftliche Sprache ist flexibel und fungiert wirksam nicht nur als Mittel zur Fixierung von Erinnerung, sondern auch als Instrument der politischen Propaganda und emphatischen Verherrlichung. Hierbei lassen sich verschiedene kommunikative Schichten differenzieren, die sich mehr vermischen als überlagern. Auf diese Weise verschiebt oder löst sich etwa die Grenze zwischen Bericht und Lob, zwischen der nüchternen Aufzeichnung eines Ereignisses, der memorialistischen und in allen Einzelheiten erzählten Erinnerung an einen Vorfall und schließlich der Verherrlichung einer Tat und ihrer Protagonisten in einem unendlich wiederholten Echo auf. Einige aufschlussreiche Beispiele mögen dies verdeutlichen.

Aus dem Jahr 1255 stammt eine sehr elegante zweispaltige Inschrift, die der Form eines offenen Buches nachempfunden wurde und heute im äußeren Bereich des Nationalmuseums Bargello in Florenz eingemauert ist. Sie kommemoriert und zelebriert (das Hendiadyoin ist hier mehr als angebracht) den Baubeginn des Palazzo del Capitano del Popolo (dann des Podestà) (Abb. 6). ${ }^{13}$

Wir befinden uns in der Regierungszeit des Primo Popolo, als die Stadt eine außerordentlich expansive Phase erlebte, auf die wir später nochmals zurückkommen werden. Zwar bestehen Zweifel über die Authentizität des auffällig qualitätsvollen Artefakts, jedoch betreffen diese nicht den eigentlichen Text. Der in Hexametern gestaltete Text, in dem unüberhörbar das Echo antiker Dichter wie Lukan, Lukrez und Claudian widerhallt, weist hinsichtlich der Komposition und des Metrums eine seltene Eleganz auf, sodass man ihn zuweilen dem Politiker und Schriftsteller Brunetto Latini zuschreiben wollte. Genannt werden sowohl der damalige Podestà Alamanno della Torre aus Mailand, welcher als vir splendens, ornatus nobilitate, urbem

13 Vgl. Gramigni 2012, 209-213 Nr. 27. 


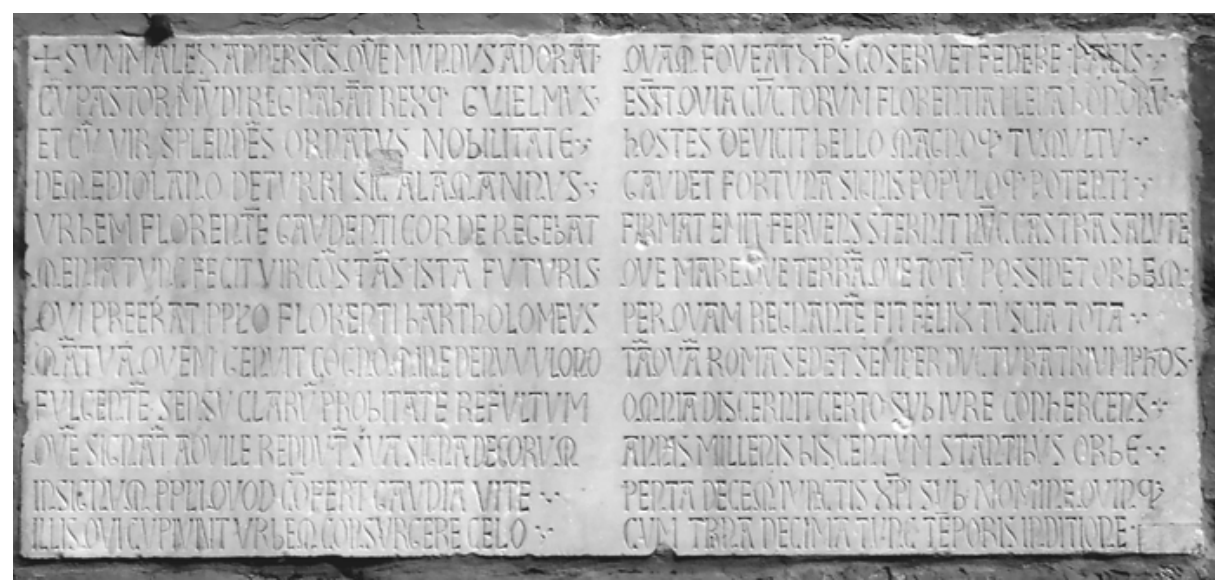

Abb. 6: Bauinschrift des Palazzo del Capitano del Popolo, heute im Nationalmuseum Bargello in Florenz. Foto aus: Gramigni 2012, 209.

florente gaudenti corde regebat, wie auch der Capitano del Popolo Bartolomeo Nuvolini aus Mantua, clarus probitate.

Gleich zwei auf das Jahr 1285 datierte Inschriften, denen jeweils das Wappen der Stadt ${ }^{14}$ bzw. des Podestà ${ }^{15}$ beigefügt ist, gedenken der Erbauung des Palazzo del Consiglio von Padua, wo sie heute noch untergebracht sind, obwohl sie ursprünglich anderswo aufgestellt waren. Beide erwähnen den Namen des damaligen Podestà Fantone de' Rossi aus Florenz, aber nur bei der ersten ist auch die Unterschrift des Werkurhebers, des magister Leonardo Bocaleca, enthalten. Ihm ist übrigens das Grab von Antenor zuschreibbar. Ebenso bewahren in Padua die Spitze und der Säulenstuhl des Kapitells einer Säule, die am Ende der Ponte Tadi aufgestellt sind (Abb. 7), die Erinnerung an den Bau der Brücke. Die Baumaßnahmen wurden mittels öffentlicher Gelder im Jahr 1300 abgeschlossen, als ein anderer Florentiner, Nicolò de’ Cerchi, Podestà war. ${ }^{16}$

Wenden wir uns nun den Toren öffentlicher Gebäude, seien sie religiösen oder profanen Charakters, zu. Als wesentlicher Bestandteil und strukturelles Element eines Bauwerks bieten sie eine Oberfläche, die für Schrift besonders geeignet ist und damit einen idealen Kontext vorgibt, um graphisch-ikonographische Programme zu realisieren. ${ }^{17}$ Die Inhalte der Nachrichten, die auf Pfosten, Architraven, Schwellen,

14 Vgl. http://cem.dissgea.unipd.it/schedarioriunito.pdf, Kartei Nr. 24.

15 Vgl. http://cem.dissgea.unipd.it/schedarioriunito.pdf, Kartei Nr. 25.

16 Vgl. http://cem.dissgea.unipd.it/schedarioriunito.pdf, Kartei Nr. 46. Hervorzuheben ist innerhalb des eher nüchternen Textes die redundante Betonung, die Brücke sei Communis Padue und das Werk für die Commune Padue realisiert worden.

17 Die zu diesem Thema vorgelegten Arbeiten sind so zahlreich, dass ich mich hier (wie auch in den folgenden Anmerkungen) auf mir unentbehrlich erscheinende Verweise beschränke: Gardner 1987, 


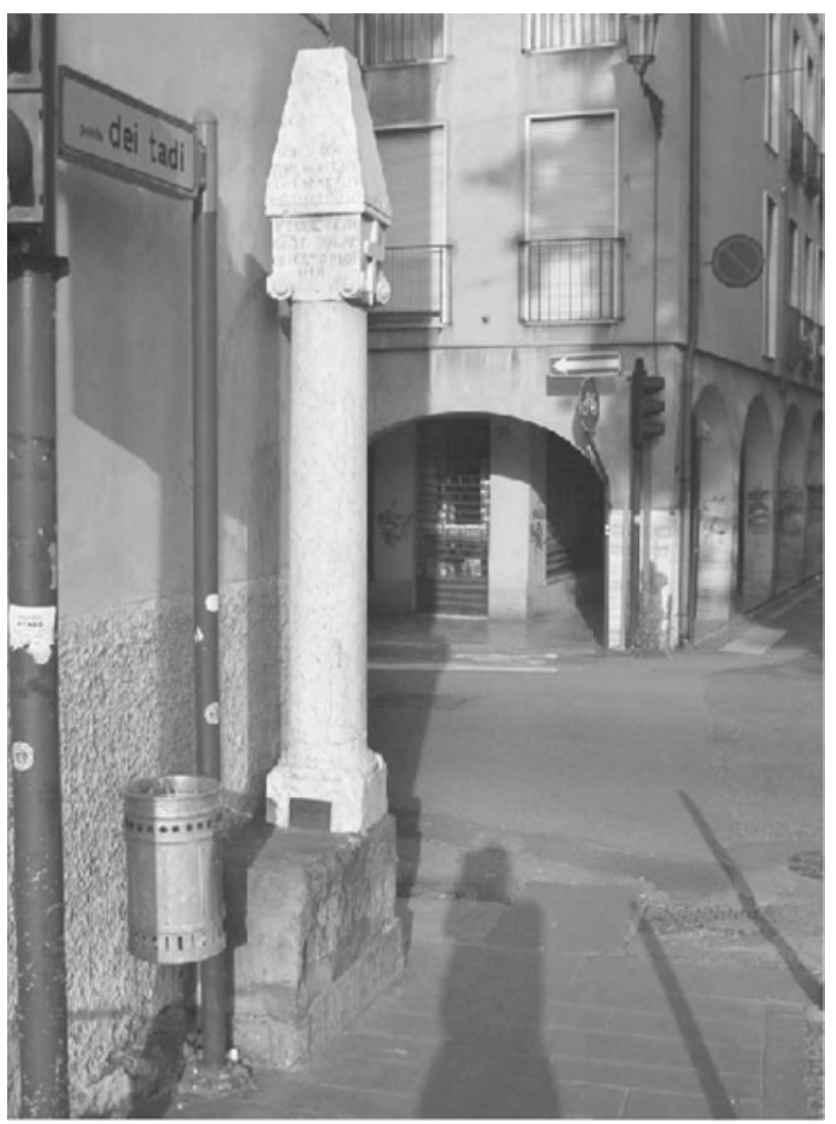

Abb. 7: Colonna an der Ponte Tadi in Padua. Foto: Verfasserin.

Tür- und Fensterflügel angebracht wurden, welche ihrerseits aus Stein oder Bronze bestehen können, sind erwartungsgemäß sehr vielfältig. In zahlreichen Fällen werden die Auftraggeber des Werkes erwähnt, in vielen anderen die ausführenden Künstler. In wieder anderen - darunter in erster Linie im Falle von Kirchentoren - drücken die am Schnittpunkt zwischen Profanem und Sakralem angebrachten Inschriften die Bedeutung vom und das Bewusstsein für den Übergang vom Materiellen ins Spirituelle aus: Die nach innen gerichtete porta materialis wird folglich zum introitus spiritualis ins Unendliche. In jeder Hinsicht nehmen die Tore sowohl in sakralen Gebäuden als auch in der bürgerlichen Architektur einen offensichtlich symbolischen Wert ein.

Favreau 1991 und Dufour Bozzo 1989, letztere mit Schwerpunkt auf der Besonderheit der Stadt Genua; Bottazzi 2012a zu Inschriften an Stadttoren in der ersten Hälfte des 12. Jh.s, insb. in Pisa, Genua, Viterbo und Mailand - Kommunen, die auch im vorliegenden Aufsatz zur Sprache kommen und denen der fortwährende Gebrauch des Kommunikationsmediums ,Inschrift‘ gemeinsam ist. 
Dies zeigt sich vor allem im Falle der Stadttore, denn die sich im Mauerring öffnenden Durchgänge stellten nicht nur einen konkreten, sondern auch einen ideellen Ort der Stadtgeschichte und Stadtordnung dar. Daher sind die städtischen Tore einer der Orte, an denen Inschriften am häufigsten zu finden sind. Diese erinnerten ursprünglich auf einfache und nüchterne Weise an die Erbauung oder Erweiterung der Tore, bis sie später dazu übergingen, regelrechte politische Botschaften auszusenden. Diese Inschriften waren sowohl an die Stadtbewohner adressiert als auch an diejenigen, die als Besucher in die Stadt eintraten und sie dabei, eben im Moment der Durchquerung des Stadttors, lasen. Darüber hinaus informierten sie häufig über die Gründe für die jeweilige Namensgebung der Tore. Dabei sind die Inschriften häufig in der ersten Person gehalten, sodass der Eindruck entsteht, das Tor spreche selbst zu dem Leser - eine Spielart die nachgerade topischen Charakter bekam.

In Ascoli Piceno finden wir den Inschriftenkomplex von der Porta di Solestà, auch Porta Cappuccina genannt. ${ }^{18}$ An der rechten Seite des Tores befindet sich eine metrische Inschrift, bestehend aus drei Hexametern, denen ein Pentameter folgt (Abb. 8). Sie erinnert an die Erbauung des Tores im Jahr 1230, die auf iussio des Podestà Fildesmido von Mogliano erfolgt sei. Die Schlussformel weist eine ungewöhnliche Art der Invokation Gottes auf, der als qui super astra manet bezeichnet wird. Oberhalb des Torbogens prangt eine anepigraphische Platte mit drei Wappen: Die zwei seitlichen sind die ältesten bekannten Wappen der Stadt Ascoli, während das Emblem im Zentrum, das bereits sehr beschädigt und folglich unleserlich ist, vermutlich dasjenige des Podestà darstellte (Abb. 9).

Was die Präsenz von Familienwappen im Kontext vieler Inschriften anbelangt, sei daran erinnert, dass es sich hierbei um einen gezielten und bewussten Einsatz der Heraldik zum Ausdruck dynastischer Zugehörigkeit handelt. Die Embleme brachten den familiären Stolz zum Ausdruck, demonstrierten aber zugleich wirksam die eigene Bedeutung und Macht. Diese Form der Selbstdarstellung und -überhöhung setzte sich nicht allzu früh, das heißt nicht vor dem 13. Jahrhundert, durch. Es ist reizvoll, wenngleich in situ kaum noch möglich, sich das Bild zahlreicher Fassaden öffentlicher Gebäude vor Augen zu führen, an denen die Wappen derjenigen hervorstachen,

18 Gut zusammengefasst bei Avarucci 1978; hinzuziehen sind die Beobachtungen von Salvi 1999, 220-226. Die Porta Solestà verwahrt das, was wir durchaus als eine kleine epigraphische Sammlung bezeichnen können. Zusätzlich zu den erwähnten „scritture esposte“ befindet sich auf der Außenseite des Tores eine Inschrift, die an die Instandsetzung eines Straßenabschnitts erinnert. Die Strecke betraf eine aus antik-römischer Zeit stammende Brücke, von der eine Abzweigung der Via Salaria, auf der ebenjenes Tor erbaut worden war, ausging. Die auf das Jahr 1283 zurückgehende Inschrift nennt den Podestà dieses Jahres, Corradino di Savignano aus Modena. Auf der Außenseite des Baus gibt es eine weitere, allerdings sehr viel spätere Inschrift aus dem Jahr 1450. Über dem Text befindet sich ein gevierter Schild mit den Wappen von Ascoli Piceno und Fermo, und der knappe Text ruft den Friedenstraktat zwischen den zwei Städten ins Gedächtnis. Die Anordnung von Bild und Text auf dieser Tafel ist bedeutungsvoll und mit Sicherheit nicht zufällig, da die Straße - wie wir wissen - bei der Brücke anfing und bis nach Fermo führte. 


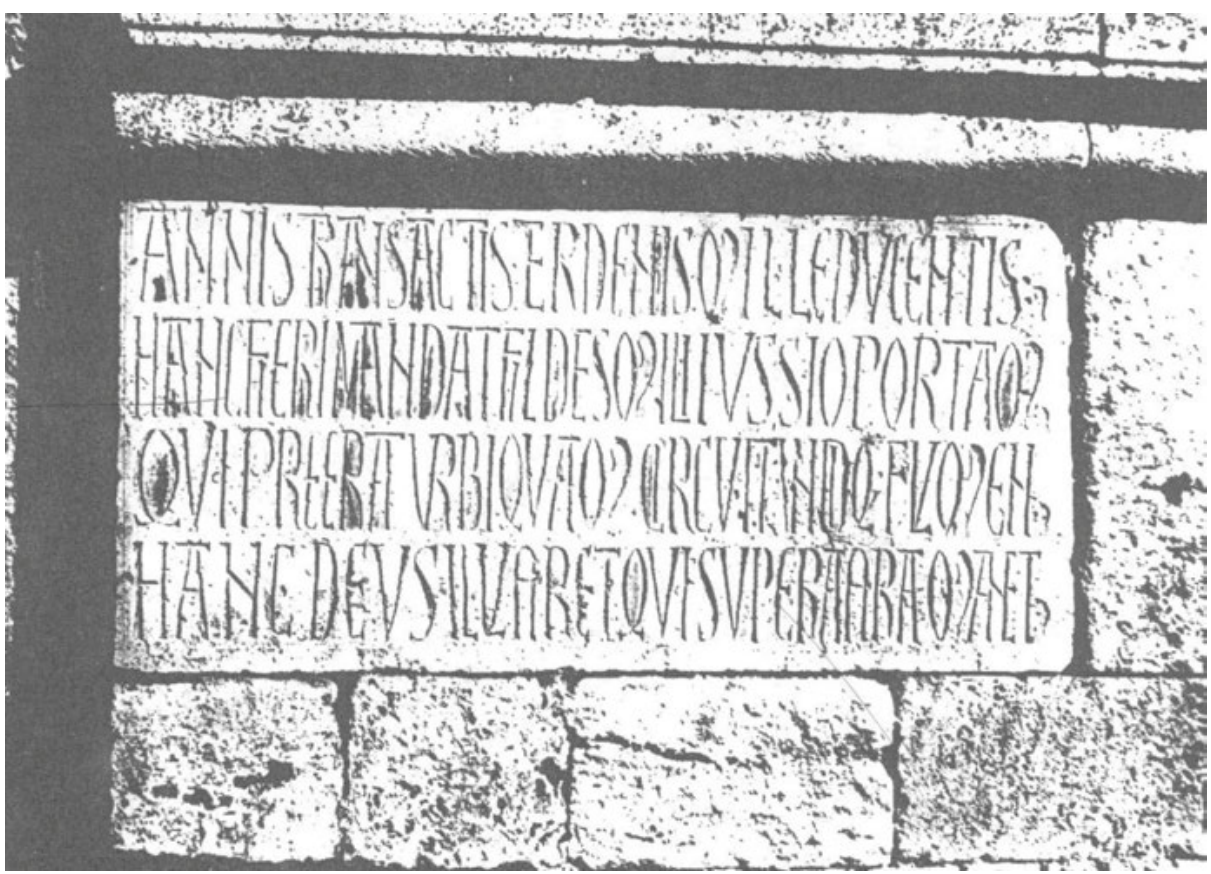

Abb. 8: Inschrift an der Porta di Solestà in Ascoli Piceno. Foto: Verfasserin.

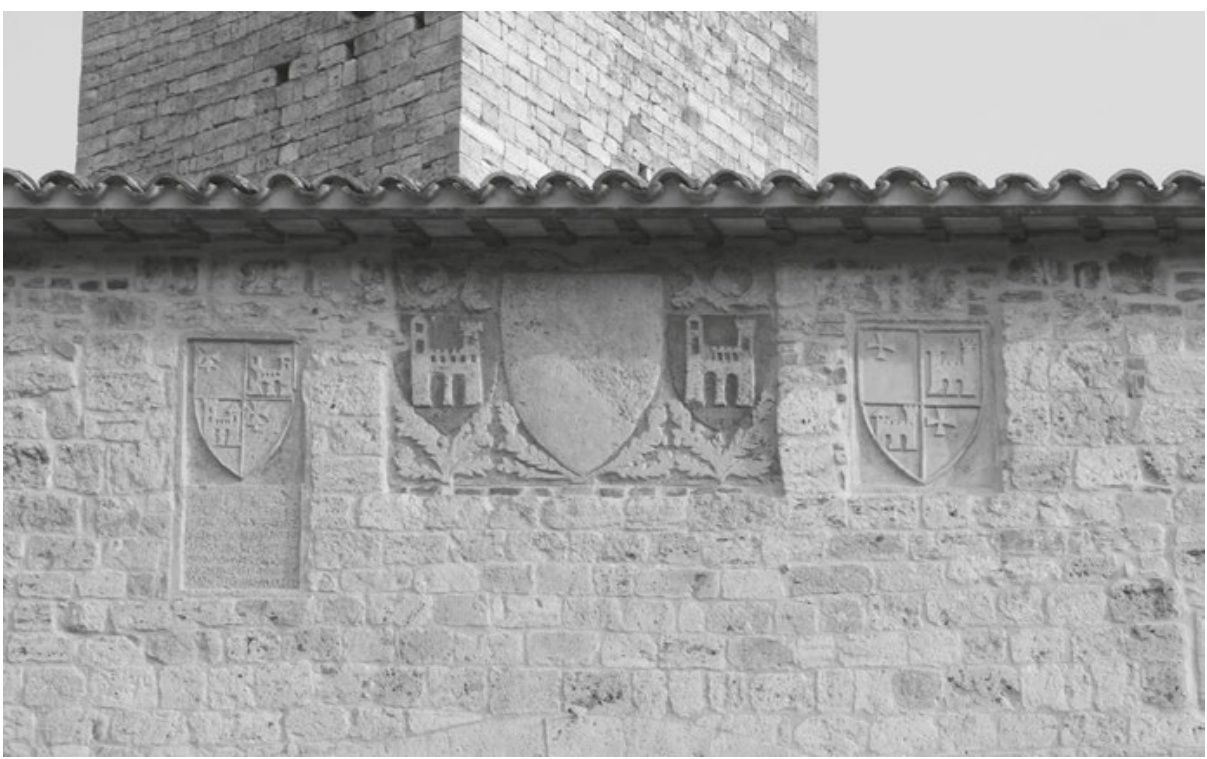

Abb. 9: Relief mit Wappen an der Porta di Solestà in Ascoli Piceno: (c) wikimedia commons. 
die ein öffentliches Amt bekleideten. Besonders waren dies das Amt des Podestà oder Capitano del Popolo.

Aber kehren wir zu den Stadttoren zurück, bei denen das Bedürfnis ihrer Erbauer nach Selbstdarstellung oftmals die Grundlage für ihre Namensgebung bildete. Wie uns die Inschriften von Viterbo berichten, kann es sich hierbei um einen Podestà handeln, der aus einer bekannten Familie stammt. Im Jahr 1255 baute beispielsweise der Podestà Bonaventura Papareschi ein Tor, die Porta Bove (Abb. 10), in Viterbo wieder auf.

Eine große Inschrift, die einmal mehr auf der Außenseite innerhalb eines Spitzenrahmens positioniert ist, lobt die Vornehmheit der Amtsperson und wünscht ihr sowie all denen, die Viterbo lieben und ehren, ewiges Leben. Außerdem ordnet sie an, das Tor möge nun zu Ehren des Podestà in Porta Bonaventura umbenannt werden. ${ }^{19}$ Die Inschrift ist von drei großen Familienwappen umschlossen. Onomastik, Toponomastik und Heraldik vermitteln, repräsentieren und bedeuten dasselbe, und zwar auf zweifache Weise: Das Tor hieß ursprünglich Porta Bove, benannt nach dem Römer Bobone di Oddone Boboni, der 1215 Podestà in Viterbo war und im selben Jahr das Tor erbauen ließ. Im Übrigen bietet Viterbo eine regelrechte Anthologie von Inschriften en plein air, welche die Gründung und Restaurierung städtischer Tore kommemorieren.

Ebenso aufsehenerregend wie berühmt ist der Fall der Inschrift der Porta Sonsa (Abb. 11). ${ }^{20}$ Es handelt sich hierbei um eine zweigeteilte Inschrift: Im ersten in Versen verfassten Teil haben wir es mit einer sprechenden Inschrift zu tun. ${ }^{21}$ Das Tor selbst erinnert an ein Privileg, und zwar an das Recht der Kommune, ihre Bürger aus der Leibeigenschaft zu befreien. Im zweiten in Prosa verfassten Teil wird die Phraseologie einer Gedenkinschriften nachgeahmt. In einer vergleichsweise nüchternen Rede in

19 Vgl. Carosi 1986, 48-51 Nr. 14. Innerhalb des Tores befindet sich eine weitere Inschrift mit doppeltem Rahmen. Die gezackte Unterseite erinnert in - von einem Prospero unterschriebenen - leoninischen amenos versus daran, dass der als neuer Hektor gefeierte Podestà Rinaldo da Brunforte den Mauerabschnitt der Porta Bove im Jahr 1290 erbauen oder restaurieren ließ: Vgl. Carosi 1986, 96-97 Nr. 33.

20 Vgl. Carosi 1986, 20-23 Nr. 4. Die Inschrift der Porta Sonsa hat Bottazzi 2006, insb. 326-340 ausführlich behandelt. In Hinblick auf ihre Datierung bereitet die Inschrift nicht unerhebliche Probleme. So ist die Datierung - im Verhältnis zur Datumsangabe in der Inschrift selbst - mit Sicherheit später anzusetzen. Bottazzi thematisiert auch die im Text vorgenommene Berufung auf das der Stadt zuerkannte Recht, ihre Bewohner zu befreien. Dieser Umstand ist als das Ergebnis einer Mystifizierung oder zumindest verzerrten Wirklichkeitsauslegung zu verstehen. Behandelt werden ferner die Funktionen, die den „scritture esposte“ von Viterbo zwischen dem Früh- und Spätmittelalter zugeschrieben wurden. Mit der frühmittelalterlichen Inschriftenkultur Italiens befasst sich allgemeiner Bottazzi 2012b.

21 In Bezug auf die sprechenden Inschriften sei auf den zusammenfassenden Beitrag von Benucci/ Foladore 2008 verwiesen. Dieser Typus von Inschriften zeichnet sich dadurch aus, dass er einen Dialog zwischen dem durch den Text synekdochisch verkörperten Artefakt und einem Gesprächspartner einleitet. Als Gesprächspartner kann sowohl das Individuum, auf das er sich bezieht (so beispielsweise der im Epitaph erinnerte Verstorbene), als auch der ideelle Leser des Textes in Frage kommen. 


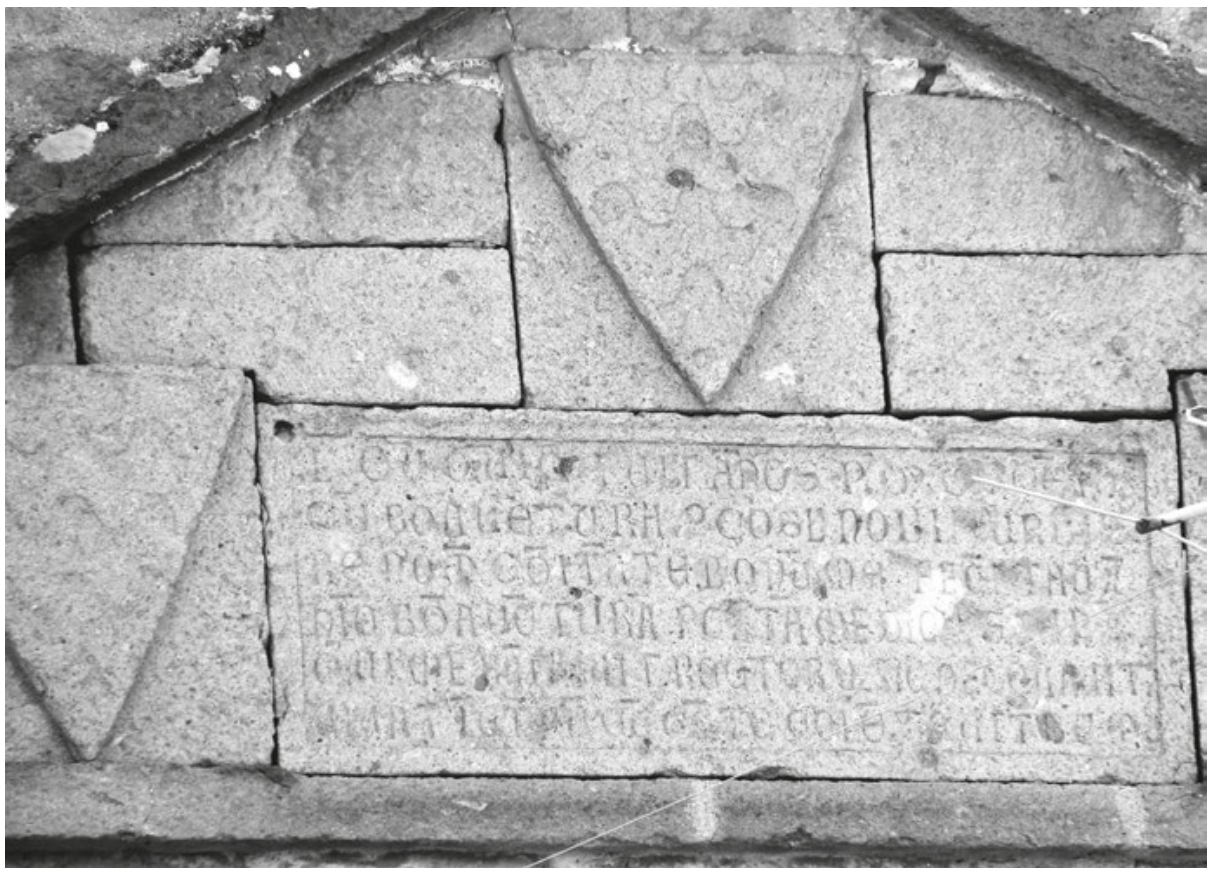

Abb. 10: Inschrift an der Porta Bove in Viterbo: Foto (c) Carlo Tedeschi.

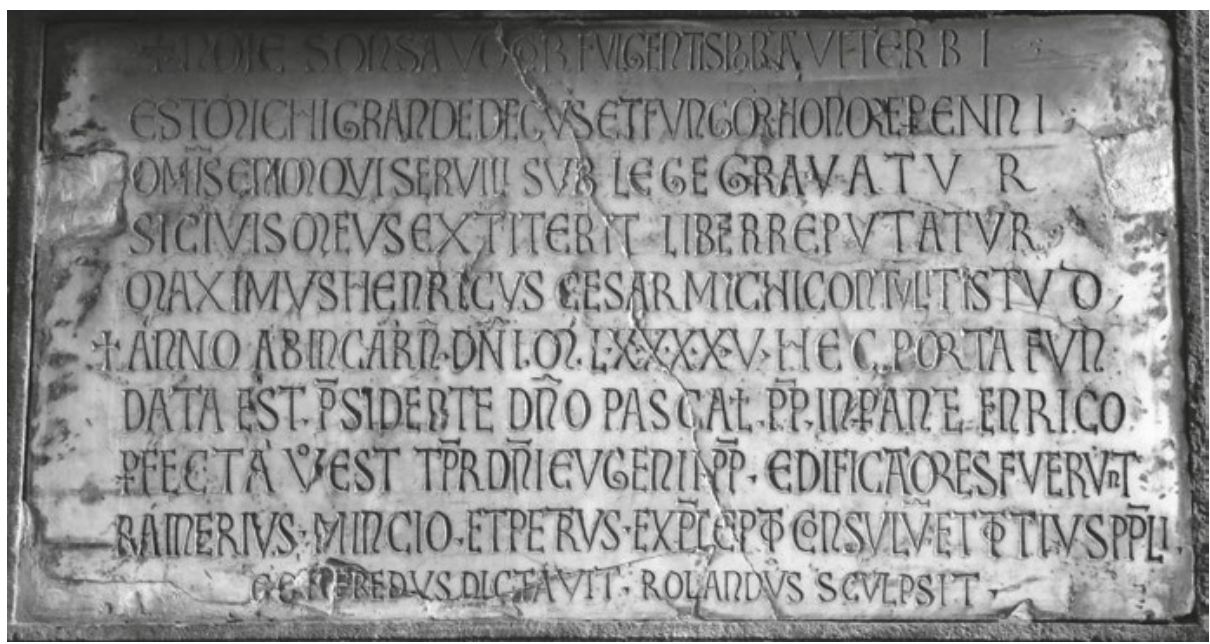

Abb. 11: Inschrift an der Porta Sonsa in Viterbo: Foto @ Carlo Tedeschi. 
der dritten Person werden die Gründung des Tores im Jahr 1095, dessen edificatores sowie der dictator und sculptor des Textes festgehalten.

Noch nüchterner ist der Text, der an den Bau der Porta S. Gallo von Florenz 1284 (Abb. 12) erinnert. Die Inschrift ist in einer gebrochenen Schrift verfasst, die weder besonders gepflegt noch sauber angeordnet ist. Die Erwähnung des Capitano del Popolo Rolandino di Canossa, nobilis miles dominus, der vermutlich im Reliefbild oberhalb der Inschrift dargestellt ist, nimmt die Hälfte des Platzes ein. ${ }^{22}$ Bezüglich der historischen Kontextualisierung des Bauwerks sei daran erinnert, dass um Florenz am Ende des 13. Jahrhunderts ein neuer Mauerring mit vier Toren errichtet wurde. ${ }^{23}$ Dies war infolge der demographischen Expansion der Stadt notwendig geworden, deren Bewohnerzahl von 50000 im Jahr 1200 auf nahezu 100000 im Jahr 1300 anstieg.

Ein weiteres Beispiel dieser Art könnte mit dem sehr bekannten und frühen Fall der mittlerweile zerstörten Porta Romana in Mailand angeführt werden. Das Tor war mit Flachreliefs und Inschriften geschmückt, die die Rückkehr der Mailänder 1167 und die Neuerbauung der Stadt und ihres Mauerrings nach 1171 feierten. Diese waren gemäß dem, was anschaulich als ,militante Stadtkunst“ bezeichnet wird, nach einem verbalen und visuellen Kommunikationssystem, einem genauen textuell-ikonographischen Programm organisiert. Der geschilderte Fall ist dermaßen anschaulich und bekannt, dass es nicht notwendig ist, bei ihm zu verweilen. ${ }^{24}$

Richten wir unseren Blick stattdessen auf die Inschrift, die an die Erbauung der Porta Torricelle in Padua erinnert, welche übrigens - da auf das Jahr 1210 datiert -

22 Vgl. Gramigni 2012, 236-238 Nr. 35.

23 Zusätzlich zum Gedenktext von 1284 war auf den Mauern eine Reihe von zwölf Inschriften angebracht, die auf die Zeit von 1310 bis 1328 datiert sind, alle in Vulgärsprache verfasst wurden und äußerst standardisierten Formeln folgen. Indem sie den Raum präzise bestimmten, fassten sie die physischen Merkmale des Befestigungsringes zusammen. Von ihnen sind nur einige Exemplare erhalten. Vgl. Pantarotto 2012, 84, die hier richtigerweise bemerkt, dass in diesen Schriften die selbstzelebrierende und kommemorierende Absicht der Auftraggeber auf den ersten Blick scheinbar nicht wahrnehmbar ist. Grund hierfür sei das Fehlen expliziter Verweise auf öffentliche Autoritäten oder jene Individuen, die für die Erbauung der Mauern verantwortlich waren: „[N]elle epigrafi fiorentine, in realtà, i committenti appaiono in ogni iscrizione della serie, ma in forma non verbale, [... bensì utilizzando dei simboli fortemente evocativi della propria identità]: si tratta del lilium e della crux, i due scudi immancabilmente presenti, alla destra e alla sinistra del testo. Facendo ricorso alla consolidata tradizione dell'araldica come mezzo di comunicazione, le lapidi sulle mura di Firenze rappresentano mirabilmente la natura del documento epigrafico in sé, come segno, anche non grafico, che si fa linguaggio. Le due insegne, visibili anche da lontano grazie all'uso del colore rosso, illustrano con evidenza anche maggiore di quello delle parole alcuni concetti fondamentali: sono espressione di una comunità unita e compatta, guidata da un'autorità che si prende cura della cittadinanza mediante la costruzione di opere difensive, imponenti e ben regolate, da mantenersi tali nel presente e nel futuro.“ 24 Im Rahmen der Konfliktereignisse zwischen den Mailändern und Friedrich I. Barbarossa waren die Zerstörung und der nachfolgende Wiederaufbau der Porta Romana epochale und äußerst symbolische Momente, die sogleich als solche verstanden und erzählt wurden. Deutlich hervorgehoben wird dies von Bottazzi 2015, die von 65-77 sowohl die Inschrift als auch die sie begleitenden Flachreliefs auf dem Zentral- und Seitenpfeiler ausführlich behandelt. 


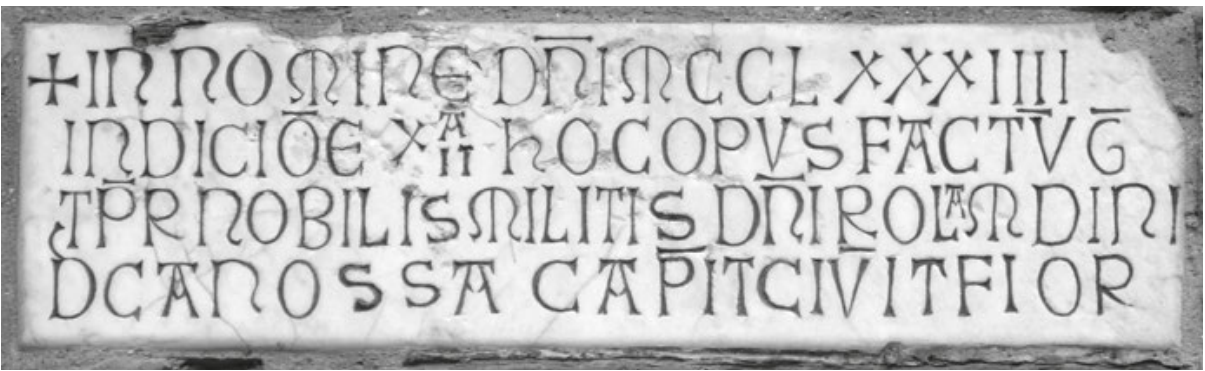

Abb. 12: Inschrift an der Porta S. Gallo in Florenz. Foto aus: Gramigni 2012, 237.

in einer nahen zeitlichen Abfolge zum vorangegangenen Beispiel steht. Die Inschrift besteht aus einem komplexen in Hexametern verfassten und etliche Anklänge an die antike Klassik beinhaltenden Text, der zum Frieden und zur bürgerlichen Eintracht aufruft (Abb. 13). ${ }^{25}$ Er wird auch in der Cronica von Rolandino da Padova zitiert. Eigentlich handelt es sich bei dieser späteren nachchronikalischen Erwähnung um eine noch komplexere und eher außergewöhnliche Angelegenheit, denn sie erlaubt uns zu verstehen, wie stark das Echo der Nachricht, deren Überbringer die Inschrift der Porta Torricelle war, im konkreten städtischen Kontexten sein konnte. Mit Blick auf die Ereignisse, die die Stadt Padua 1236 erschütterten, bemerkt Rolandino nämlich betrübt, dass zu seiner Zeit jenes eintrat, was der Autor des Inschriftentextes, der Grammatiker und Dichter Giovanni Valdetaro - auch an den damaligen Podestà Giacomo Andito aus Piacenza erinnernd -, prophetisch oberhalb der Porta Torricelle geschrieben hatte: arboreis frustra petitur sub frondibus umbra,/ interius morbus si viscera torret acutus. ${ }^{26}$

Öffentliche Einrichtungen, Mauern und Brücken erinnerten die italienischen Stadtbürger an ihren jeweiligen Erbauer: Der Brauch, Baumaßnahmen durch auf Steine geschriebene Texte festzuhalten und zu dokumentieren, erlangte somit einen immer weiteren Anwendungsbereich, wodurch wiederum eine Praxis der Schriftnutzung geschaffen bzw. gefestigt wurde. Die Stadtmauern stellten einerseits ein konkretes sowie ein metaphorisches Instrument zur Definierung der Physionomie, Kohäsion und Individualität urbaner Zentren dar; sie besitzen die Aufgabe, ihre Gemeinden zu trennen wie zu verteidigen, zu versammeln und zu vereinen; sie fassen die städtische Identität zusammen und stellen sie gleichsam nach außen zur Schau. Andererseits bieten sie aufgrund ihrer Struktur und Bedeutung einen idealen und weiten graphischen Raum, der frei zur Verfügung steht und sich bestens dafür eignet, Schrift aufzunehmen. Dies erfolgt gemäß einer seit der klassischen Zeit tief verwurzelten Gewohnheit, die sich ab dem 12. Jahrhundert zweifellos wieder intensivierte.

25 Vgl. http://cem.dissgea.unipd.it/schedarioriunito, Kartei Nr. 47.

26 Vgl. Fiorese 2004, 148-149 und 596 Nr. 57. 


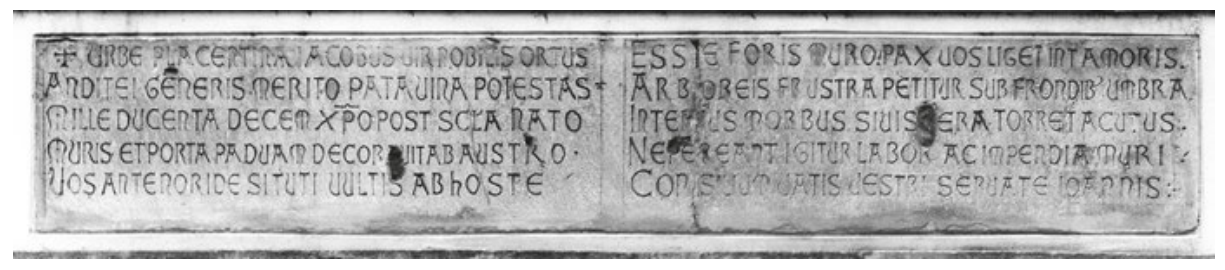

Abb. 13: Bauinschrift der Porta Torricelle in Padua. Foto: Verfasserin.

Als Gründe hierfür sind der steigende Alphabetisierungsgrad der Stadtbevölkerung und die hiermit in Zusammenhang stehende Diversität an Funktionen zu nennen, die der inschriftlichen Kommunikation im Stadtraum zukam. Ferner spielte die damalige Intensivierung der Wiederinstandsetzung, Erweiterung oder Neuerbauung von Mauerringen und den mit ihnen verbundenen Verteidigungsstrukturen eine wichtige Rolle. Wie wir bereits feststellen konnten, korrelierte das Wachstum baulicher Aktivitäten eng mit dem ökonomischen und demographischen Wachstum der italienischen Kommunen. ${ }^{27}$ In die Mauern wurden die Namen derjenigen eingraviert, die sie ideell und konkret errichtet hatten. Genannt wurden also die institutionellen Auftraggeber ihrer Erbauung (wie etwa Konsuln, Podestà, Capitani del Popolo) sowie die artifices und magistri, die am Bau tätig waren. Außerdem wurden die Begebenheiten, die zur Erbauung der Mauern geführt haben bzw. die Eingriffe zur Instandhaltung derselben erwähnt. Neben dem Gedenken an ein Ereignis drängt sich im kommunalen Italien die Erinnerung an dessen Protagonisten auf, worunter auch diejenigen zu zählen sind, die den epigraphischen Text oftmals mit hoher Geschicklichkeit und großem Wissen verfasst hatten. ${ }^{28}$

Zuweilen konnten die Inschriften auch eine recht einfache, ausschließlich ortsnamenkundliche Funktion besitzen, wie es etwa bei den Inschriften der Fall ist, mit denen die Bergfriede bezeichnet wurden. Als Beispiele ließen sich die Verteidigungstürme innerhalb des aus dem Jahr 1326 stammenden dritten Mauerrings von Bologna

27 Einige hilfreiche Anregungen zu einer allgemeinen Einordnung dieses Phänomens mit Blick auf die gesamte italienische Halbinsel finden sich bei Ulianich/Vitolo 2001 und Panero/Pinto 2009.

28 Anregend sind diesbezüglich die Überlegungen von Guerrini 2013. Sie erfasst die vielfältigen Typen der auf dem Mauerring lesbaren Nachrichten und bemerkt auf S. 2: „Nel mondo romano le epigrafi delle mura hanno un carattere più standardizzato, essenzialmente celebrativo-dedicatorio. Tali attestazioni iscritte nel medioevo sono pertinenti a più tipologie; a fronte di epigrafi relative alla memoria pubblica esposta di un intervento di un rappresentante del potere, la maggior parte, si hanno anche epigrafi di tipo didascalico, di tipo encomiastico celebrativo, attributive, che menzionano la committenza, o ancora epigrafi che sfruttano il supporto ,mura' per la sua valenza di monumento forte, visibile, rappresentativo della città, per celebrare eventi non strettamente connessi con la struttura stessa, come nel caso dell'epigrafe di Porta Aurea a Pisa. L'epigrafe si qualifica quale luogo della memoria non solo di opere concrete ma anche di azioni belliche e/o politiche. Non mancano poi anche sigle che documentano dell'organizzazione del lavoro di cantiere e della circolazione delle maestranze“. 
anführen. Die Türme wurden nach der Cappella, also nach jenem Regierungsbezirk benannt, der sie errichten ließ. Ebenfalls im Verteidigungsring von Bologna angebracht, feiert eine Inschrift aus dem 14. Jahrhundert die Initiative zur Erbauung zweier Bögen, die hier nicht bei einer öffentlichen Autorität, sondern beim Bürger Giovanni Casari lag. Wie es die Inschrift feierlich und erhaben zum Ausdruck bringt, handelte dieser ex mera liberalitate et de propria facultate, jedoch vor allem utilitati proprie semper sacram rem publicam anteponens. ${ }^{29}$

\section{Inschriften innerhalb des Stadtraums (und ausserhalb)}

Die anhaltende Notwendigkeit im italienischen Spätmittelalter, „scritture esposte“ als materiale Ausdrucksformen der öffentlichen Sache zu verwenden, trug dazu bei, dass inschriftliche Texte auch auf kleineren Denkmälern Eingang fanden - womit eine in der Antike wurzelnde Tradition wiederaufgenommen wurde. Die Rede ist beispielsweise von Straßensäulenstümpfen oder Grenzsteinen. Das nun schon häufiger zur Sprache gebrachte Bologna liefert einen exemplarischen und vieluntersuchten Fall für die reiche Produktion öffentlicher Inschriftlichkeit. Dort ist unter anderem die Verwendung einer gelungenen Zusammenstellung aus Bild und geschriebenem Wort feststellbar, die insbesondere topographisch-toponomastische Funktionen besitzt. ${ }^{30}$ In Bologna sind häufig Gewohnheiten zu beobachten, die andernorts zwar ebenfalls bekannt waren, aber sich nur hier in einer solch großen Anzahl an Zeugnissen niedergeschlagen haben. Sie bekunden vor allem einen kontinuierlichen Umgang mit dem öffentlich ausgestellten Wort, das offenbar als natürliches Element des Stadtalltags und des städtischen Weichbilds erlebt wurde. Ihm wurde die zeitliche Festlegung orts- und personennamenkundlicher Informationen anvertraut.

Wenden wir uns zuerst den Straßenkreuzen zu, also jenen Kreuzen, die ursprünglich an Knotenpunkten der äußerst begrenzten frühmittelalterlichen Mauerringe lokalisiert waren. ${ }^{31}$ Von diesen sind vier erhalten geblieben, die erstaunlicherweise unterschiedliche Chronologien aufweisen. Die Praxis, diese gemeißelten und ,spre-

29 Vgl. Breveglieri 1989, 420-422.

30 Vgl. Breveglieri 1989, passim, der in Bezug auf das Bologna der kommunalen Zeit unterstreicht: „[L]'uso di documentare con pietre iscritte le imprese costruttive raggiunse un campo d'applicazione estesissimo, creando nei cittadini [...] una fortissima assuefazione alla scrittura“ (ebd., 410-411). Über viele der öffentlich ausgestellten Inschriften aus Bologna, die als stolzer Ausdruck des Selbstbewusstseins und der städtischen Autonomie interpretiert werden, äußert sich Pini 1999, passim. Verwiesen sei ferner auf den jüngsten Beitrag von De Rubeis 2016, die sich unter anderem mit der Inschriftenproduktion in Bologna und Padua in spätmittelalterlicher Zeit befasst.

31 Die in die Kreuze von Bologna gemeißelten Inschriften wurden von Gozzadini 1863, $42-47$ abgeschrieben und ediert. Eine aktuellere Untersuchung hierzu bietet u. a. Bussolati 1988. 
chenden' Kreuze an besonders stark frequentierten Orten wie Hauptverkehrskreuzungen zu verorten, zeigt sich im Falle der sogenannten Croce del Mercato: das Kreuz wurde 1219 auf der Spitze einer Marmorsäule aufgestellt, um an die Errichtung des neuen Marktplatzes und einiger Mühlen am nahegelegenen Kanal zu erinnern. Allerdings konnten Straßenkreuze auch mit der Notwendigkeit verbunden werden, die Physionomie und Erkennbarkeit eines Ortes zu definieren und zu vereinfachen.

In Padua sind von einer Reihe von Grenzsteinen noch drei Exemplare erhalten, die weniger als einen Meter hoch sind und die Grenzen der campanea markierten, also eines unmittelbar an die Stadt angrenzenden Gebietsstreifens, in dem die städtische Verwaltung direkte Gerichts- und Fiskalkontrolle ausübte. ${ }^{32}$ Diese Grenzsteine wurden 1286, zwei Meilen vom Palazzo del Comune entfernt, entlang aller Hauptstraßen aufgestellt. Insbesondere ein Grenzstein, der auf den 16. Oktober datiert ist, weist neben dem Namen des Podestà (Barone de' Mangiatori von S. Miniato) eine symbolische invocatio zu Textbeginn auf. Die zwei unteren Seiten des Grenzsteins zeigen das Stadtwappen von Padua, das als Flachrelief innerhalb eines Schildes ausgeführt ist.

\section{Weitere Beispiele: Von Genua nach Cagliari}

Wenden wir den Blick nach Genua, das an bekannten und bedeutenden Zeugnissen reich ist, wobei sich diese in vielen Fällen von den bisher behandelten deutlich unterscheiden. Hierzu sind die Arbeiten von Rebecca Müller grundlegend. ${ }^{33}$ Im genuesischen Bestand öffentlicher Inschriften sind Gedenksteine zur Errichtung und Erweiterung öffentlicher Bauwerke wie etwa der Mauern und Stadttore, des Hafens und der Fontane Marose - um nur die bedeutendsten Monumente zu nennen - im Überfluss vorhanden. Wir finden insbesondere Zeugnisse für die eindrucksvolle Verbindung von Schrift und Bild. In der Gesamtheit der bildlichen Kompositionen wirkt die Schrift zuweilen wie ein dem Bild untergeordnetes Element, wohingegen sie sich in anderen Fällen auch von diesem befreien kann, es mitunter sogar beherrscht. Dies ist in der Gruppe von Inschriften und Flachreliefs der Fall, die über einen sehr wichtigen genuesischen Sieg über Pisa im Jahre 1290 informieren, als die Genuesen den Porto

32 Vgl. Benucci 2015, 55-57 Nr. 3, 69-71 Nr. 6 und 247-249 Nr. 60. In der Steinsammlung des paduanischen Museo d'Arte medievale e moderna ist auch ein weiterer Grenzstein, der des districtus Padue aus dem Jahr 1309, aufbewahrt (ebd., 72-74 Nr. 7), der sich in seiner Form von den Exemplaren des vorangegangenen Jahrhunderts unterscheidet. Da der Grenzstein nach oben verjüngt und mit einem Tympanon versehen ist, ist er in Hinblick auf die Textanordnung, die den oberen Bereich des Säulenstumpfes vollständig einnimmt, zwingend: Der Text kann nichts Anderes tun, als die damaligen Zeitkoordinaten wiederzugeben, indem er zusätzlich zum Jahr den Namen des amtierenden Podestà Ermanno di Corrado della Branca aus Gubbio erwähnt. Die Stadtwappen befinden sich hingegen in einem spitzbogigen Schild auf den Dachschrägen des Tympanons.

33 Müller 2002. 
Pisano zerstörten und sich der Sperrketten bemächtigten, die zu dessen Verteidigung dienten. ${ }^{34}$

Der Erfolg dieses Unternehmens wurde einem Mitglied der Doria, dem capitaneus Corrado, zugeschrieben. Konkret geht es um vier Inschriften, von denen zwei in der Kirche von S. Matteo, der Familienkirche der Doria, aufbewahrt werden (Abb. 14). Eine dieser Inschriften, die an der Fassade von S. Matteo angebracht ist, bietet bloß einen knappen Text. Die anderen drei hingegen sind Kopien ein und derselben Vorlage und integrierender Bestandteil einer Reliefplatte, welche die Hauptelemente der Hafenanlagen des Porto Pisano abbildet. Die Platte zeigt auch jene Sperrketten, welche die Genuesen in ihre Stadt verlegten und - wie es scheint - neben einer dieser Inschriften, die ursprünglich auf der Außenseite einiger städtischer Gebäude verortet waren, zur Schau stellten. Die Benutzung dieses besonderen Verfahrens zur Verherrlichung eines wichtigen Ereignisses für das Stadtleben zeigt sich in Genua auch anhand weiterer, analoger Fälle. Gemeint sind die oberhalb und unterhalb der venezianischen Löwen eingravierten Inschriften, die an die Eroberungen von Pola und Triest 1380 erinnern. ${ }^{35}$ Wie sie explizit und stolz festhalten, handelt es sich bei den Löwen um eine Kriegsbeute, da sie aus militärisch eroberten Städten stammen.

Doch kehren wir noch einmal zum außergewöhnlichen Inschriftenkomplex von Piazza S. Matteo zurück. Hier verlaufen die Inschriften entlang der Fassade der gleichnamigen Kirche und des Palazzo von Lamba Doria. Indem sie - einem offenen Buch gleich - die Pracht der Doria dem Blick des Betrachters anbieten, erzählen sie gleichsam von ihr. Es handelt sich hierbei um einen graphisch-architektonischen Komplex von hoher Wirkungskraft, in dem die Schrift zum monumentalen und gleichzeitig - in Hinblick auf die Lobpreisung der erinnerten Ereignisse und Personen - funktionalen Element wird. Auf fünf der weißen vorspringenden Streifen, die die Kirchenfassade gliedern, sind Inschriften eingraviert, die militärische Siege der Doria zwischen dem 13. und 16. Jahrhundert verherrlichen. Unter anderem schildern sie den Sieg von Meloria 1284 unter Oberto Doria, die bereits erwähnte Zerstörung des Porto Pisano, den 1298 unter Lamba erlangten Sieg von Curzola, Paganos Siege von Konstantinopel und Porto Longone 1353 und 1354 und schließlich den Sieg bei Pola unter Luciano im Jahr 1379. Die Verherrlichung von Lamba Doria wird durch eine Inschrift auf der Fassade seines Palazzo zusätzlich verstärkt, die der Kirche gegenüberliegt und mit geringfügigen Abweichungen den Text der Inschrift von S. Matteo wiederholt. Lambas Erhöhung wird ferner durch dessen Sarkophag akzentuiert, der sich an der Außenwand der Kirche oberhalb der Gedenkschrift befindet und von einem Grabstein

34 Die erste der drei Inschriften, die von einem Flachrelief begleitet werden, ist im Museo di S. Agostino in Genua aufbewahrt. Die zweite ist im Kreuzgang der Kirche von S. Matteo zu finden. Die dritte befindet sich auf dem Friedhof von Pisa: Vgl. Origone/Varaldo 1983, 44f. Nr. 10; Silva 1987, 171-172 Nr.124, und I litorali toscani e la Maremma 1982, 36. Zur anikonischen Inschrift auf der Fassade von S. Matteo vgl. Silva 1987, 171 Nr. 123.

35 Vgl. Silva 1987, 35 Nr. 58 und 106 Nr. 185. 


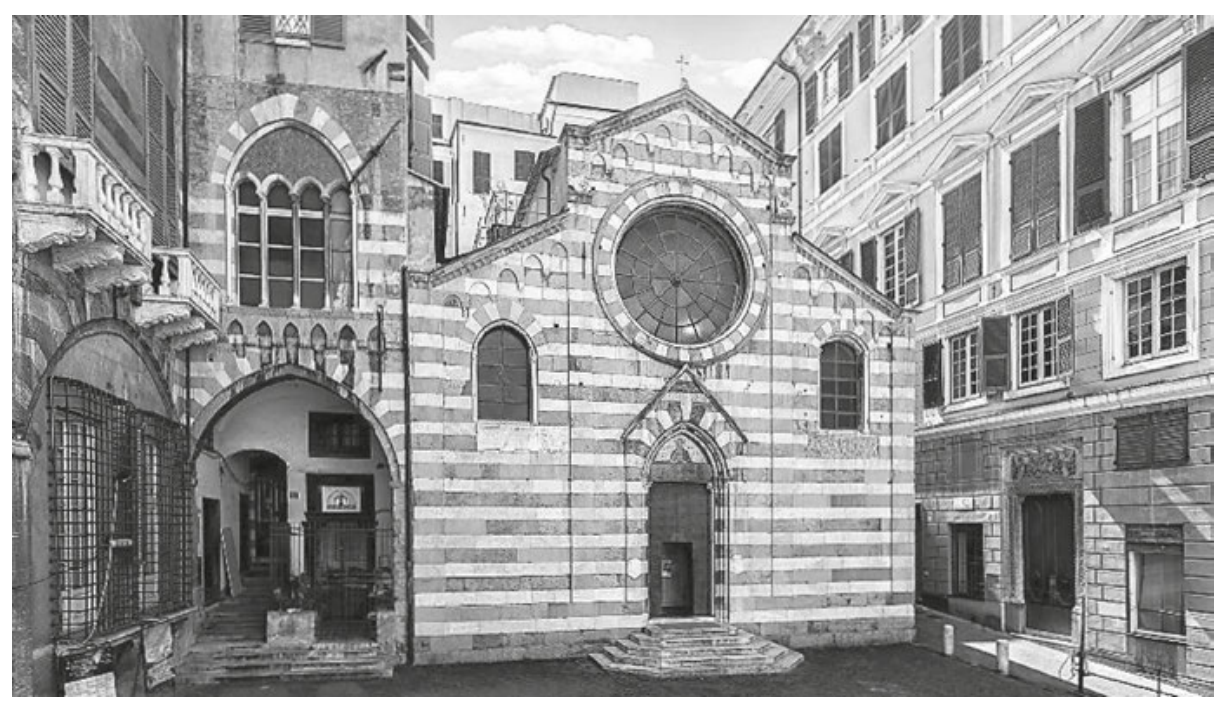

Abb. 14: Kirche S. Matteo in Genua. Foto: Verfasserin.

begleitet wird. ${ }^{36}$ Die Verwendung der Schrift als das komplementäre Element einer architektonischen Struktur, wie es das Beispiel von S. Matteo abbildet, wurde auch in der Kirche von S. Stefano nachgeahmt. Diese bewahrt in ihrer nach dem üblichen Zweifarbenmuster der genuesischen Romanik gegliederten Fassade zahlreiche Inschriften. Sie sind in die weißen vorspringenden Streifen eingraviert, reichen chronologisch vom 12. bis zum 16. Jahrhundert und zelebrieren den Prunk einer weiteren bedeutenden Familie der Stadt, der Da Passano (Abb. 15).

Die bisher erwähnten Fälle, die nur einige Beispiele unter vielen weiteren interessanten darstellen, sind zugegebenermaßen nicht außergewöhnlich und eher vorhersehbar. Doch eignete sich die inschriftliche Kommunikation auch Themen an und trat in Kontexte ein, die unvorhersehbar sein konnten. Hierin zeigen sich zusätzlich ihre Dynamik, die Fähigkeiten derjenigen, die ihr Potential am besten nutzten, und gleichzeitig die Vertrautheit, welche die Stadtbevölkerung mit der Zeit im Umgang mit „scritture esposte“ erlangte. So erstaunt, auch wenn nur bis zu einem gewissen Grade, das Phänomen der sogenannten chartae lapidariae. ${ }^{37}$ Diese sind bereits für das Frühmittelalter belegt, kommen im kommunalen Zeitalter aber besonders häufig vor. Ohne den Sachverhalt zu sehr zu vertiefen, erinnere ich zum Zwecke meines Themas bloß daran, dass es sich dabei um Inschriften handelt, die Urkunden regel-

36 Die beachtliche Inschriftensammlung von San Matteo ist bei Silva 1987, 69-90 Nr. 121-156 ediert. Die erwähnten Inschriften befinden sich in der Reihenfolge ebd., 70-74 Nr. 122-126, 77 Nr. 132, 78 Nr. 134 und 81 Nr. 139.

37 Siehe hierzu ausführlich den Beitrag von Vincent Debiais in diesem Band sowie den zusammenfassenden Beitrag von Banti 1992. 


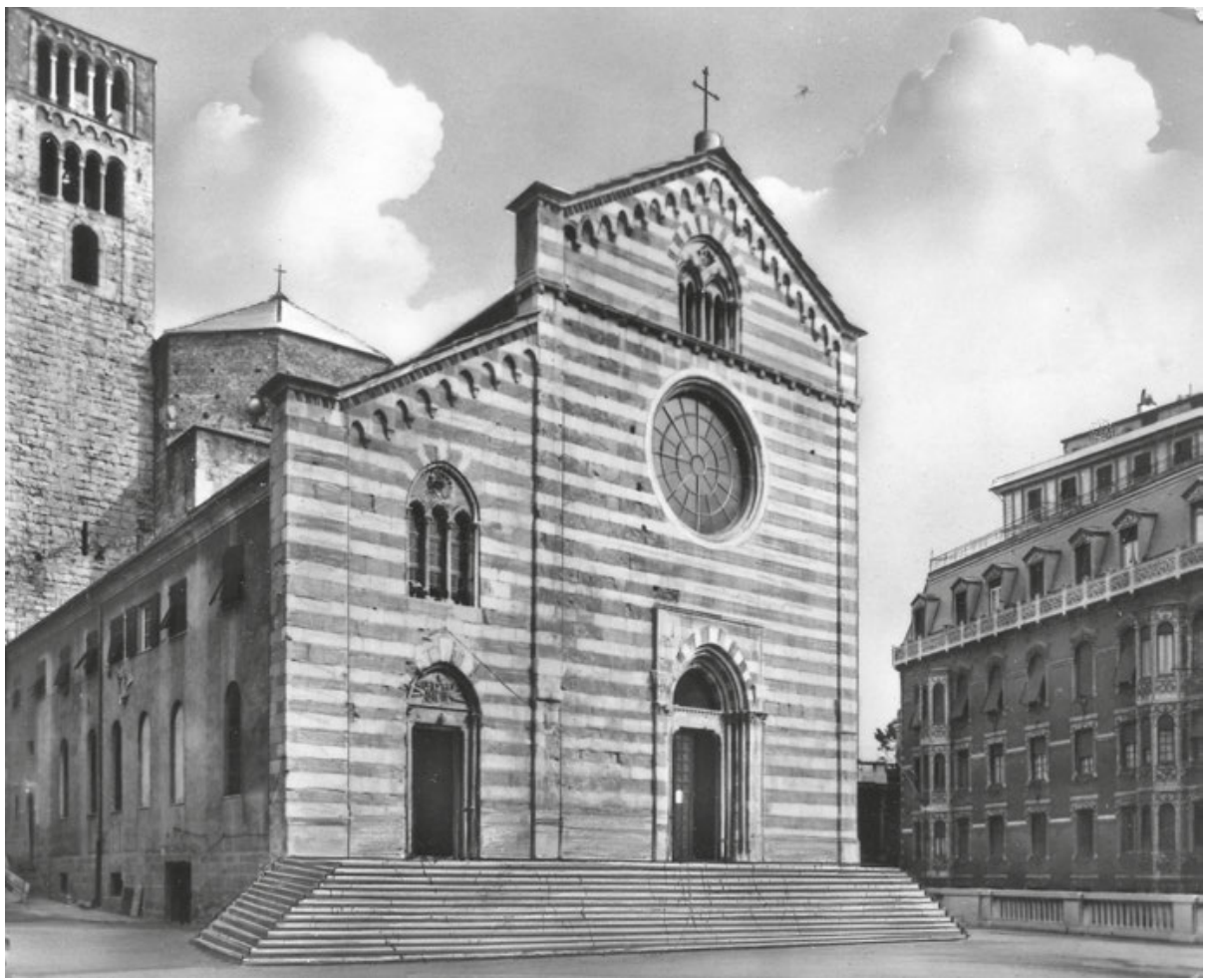

Abb. 15: Kirche S. Stefano in Genua. Foto: Verfasserin.

recht abbilden, seien diese öffentlich oder privat. Sie zeigen alle formalen Merkmale von Dokumenten, die an Kanzleien bzw. von Notaren ausgestellt wurden. Hierdurch verkörpern sie das, was in der antiken Epigraphik als „eingraviertes Recht“38 bekannt ist. Dieser Typologie gehören Steindekrete an, also auf Stein übertragene gesetzliche Bestimmungen, die dem Wunsch nachkamen, eine genaue Vorschrift oder einen gültigen Beschluss erga omnes durch ihre Materialisierung in einem inschriftlichen Text zu verbreiten. Was ihre Inhalte anbelangt, so besitzen sie in der Regel eine allgemeine Bedeutung für die Stadt, da sie Fragen des politischen Lebens behandeln und oftmals in Verbindung mit Statutentexten stehen, deren Bestimmungen sie übernehmen. Allgemein berichten uns diese Inschriften nicht nur von den Personen, die öffentliche Ämter bekleideten, sondern auch von der Entwicklung kommunaler Institutionen. Manchmal lassen sie uns auch an Konflikten zwischen unterschiedlichen Stadtparteien teilhaben.

38 Wir übernehmen an dieser Stelle den zutreffenden und einprägsamen Ausdruck von Licandro 2002. 
Dies ist etwa bei der bekannten petra iustitiae von Perugia aus dem Jahr 1234 der Fall, die zur Amtszeit des aus Bologna stammenden Podestà Ramberto Ghislieri gefertigt wurde. Nebenbei sei erwähnt, dass hierfür eine Inschrift aus antik-römischer Zeit wiederverwertet wurde. Der Inschrifteninhalt ist zweigeteilt: Im ersten Teil wird die Tilgung sämtlicher Stadtschulden erklärt, während im zweiten die Fälle aufgezeigt werden, in denen eine Besteuerung verlangt werden soll. Anschließend finden wir diesen Text in den Stadtstatuten von $1279 .{ }^{39}$

Der Fall der Inschriften, die auf der Südseite des Doms von Ferrara angebracht sind, ist einzigartig, jedoch auch schwierig zu klassifizieren. Die Inschriften geben einen wichtigen Teil dessen wieder, was wir als eine Art von Stadtstatut bezeichnen können. ${ }^{40}$ Meines Wissens nach lässt sich andernorts keine vergleichbare Marmorplatte finden. Sie beinhaltete das eingravierte Statut, das vom Consiglio dei Sapienti am 13. Mai 1173 verabschiedet und durch die Volksversammlung unter Eid bestätigt wurde. Zunächst waren die Inschriften aus Ferrara im unteren Bereich des Mauerwerks verortet und somit für jeden, der vorbeilief, sichtbar. Mitte des 14. Jahrhunderts verschwanden sie dann endgültig aus dem Blickfeld, als die hölzernen Stände der Kurzwarenhändler, die seit Ende des 13. Jahrhunderts an der Kirche lehnten, in den dreißiger Jahren des 14. Jahrhunderts zu gemauerten Geschäften umgebaut wurden. Vielleicht erfolgte diese Umstrukturierung nicht zufällig zeitgleich mit dem unaufhaltsamen Aufstieg der Familie d'Este und somit mit dem Ende der freien Kommune. Besondere Beachtung verdient die Wahl des Doms als Ausstellungsort, denn dieser ist durchaus als Gebäude des Volkes und Zentrum des Stadtlebens zu verstehen. Dies steht im Übrigen gänzlich mit den Bestimmungen des Textes im Einklang, in denen eine Vereinbarung zwischen dem Bischof und der Stadt bestätigt wurde. Die Wahl der Aufstellungsorte von Inschriften öffentlichen Interesses scheint im Allgemeinen nicht zufällig getroffen worden zu sein, sondern sie war wohlüberlegt, bewusst und berücksichtigte die vielen Dreh- und Angelpunkte des politischen Stadtlebens.

Die mittelalterlichen Städte berichteten von ihrer Geschichte und den identitätsstiftenden Ereignissen, die sie voneinander unterschieden. Sie (re)konstruierten Geschichte, indem sie deren Synthese in Inschriften zur Schau stellten, die sie an äußerst symbolischen Orten in der Stadt positionierten. Wie wir für Genua festgestellt haben, konnten Kirchen- und Palastfassaden zu den Seiten eines „Kollektivgedächtnisbuches“ werden. In diesem Zusammenhang stellt Pisa wohl den bekanntesten und frühesten Fall dar. Hierüber werde ich nichts anmerken, um nicht das zu wiederholen, was schon seit geraumer Zeit von Anderen erschöpfend dargelegt worden ist. ${ }^{41} \mathrm{Jedoch}$

39 Vgl. Bartoli Langeli 1983, 313-315 Nr. 145.

40 Vgl. Franceschini 1969 und 1972, 101-108.

41 Pisa hat schon immer die Aufmerksamkeit von Gelehrten auf sich gezogen. Dieser Umstand ist in der stets hervorgehobenen Besonderheit ihrer Inschriftenproduktion im öffentlichen Bereich begründet, die deutlich politische Funktionen besaß. Verwiesen sei hier zuerst auf die Arbeiten von Giuseppe Scalia, insb. Scalia 1963 und 1972. Daneben ist die gesammelte Anthologie von Banti 2000 
sind die Inschriften, die epochale, für die Stadtgemeinschaft bedeutende Ereignisse feiern, zahlreich. Hierunter sind zum Beispiel die Friedensverträge zu nennen, die die wiederhergestellte Eintracht zwischen verfeindeten Parteien rühmten, bis hin zu den Abkommen, die zwischen gegnerischen Städten geschlossen wurden. Die Inschrift, die sich ursprünglich auf dem Palazzo del Capitano del Popolo von Viterbo befand, mag dies eindrücklich veranschaulichen. Sie ist auf das Jahr 1275 datiert und erinnert daran, wie der Podestà Pandolfo, Graf von Anguillara, und der Capitano del Popolo Rolando degli Alessandri discordes mira et sollepni pace [pacaverunt]. ${ }^{42}$ Am selben Ort und mit demselben Datum fand sich eine weitere Inschrift, die - in Reimversen verfasst - noch einmal Pandolfo und Rodolfo erwähnte, hier aber in ihrer Rolle als Bauherren des Palazzo del Popolo. Wie im Inschriftentext angemerkt wird, ${ }^{43}$ wurde die Inschrift von einem Löwen begleitet, der das Stadtwappen bildete. Er befand sich offenbar direkt neben der Inschrift.

Ein weiterer Fall, der in gewisser Weise auch eine pisanische Prägung besitzt, stellen einige Inschriften aus Cagliari dar, insbesondere zwei Zwillingsinschriften, die das Gedenken und die Lobpreisung mit ausgesprochen dokumentarischen Aspekten verbinden oder - besser formuliert - verschmelzen. ${ }^{44}$ Es handelt sich hierbei um die Inschrift von 1305 am Sockel der Torre von S. Pancrazio und die von 1307 auf der Torre dell'Elefante, zwei zur Verteidigung der Tore errichtete Türme im Mauerring der Stadt, den die Pisaner zu Beginn des 14. Jahrhunderts erbauten. ${ }^{45}$ Beide Inschriften preisen die von der Stadt Pisa betriebene Errichtung des Bauwerks. Dieser Umstand lässt sich dadurch erklären, dass Cagliari, oder vielmehr das von den Pisanern errichtete und gefestigte Stadtviertel Castel di Castro, Pisas Herrschaftsbasis auf Sardinien bildete. Die Inschriften sind metrisch und in Versreimen verfasst. In beiden werden nicht allein die Namen der Burgherren genannt, also der zwei höchsten Amtspersonen, die von den Stadtbürgern gewählt wurden, sondern auch die des Baumeisters, des Notars und des Turmarchitekten Giovanni Capula - womit politische Personen mit der Berufsgruppe, die das Werk konkret realisiert hat, auf die gleiche Ebene gesetzt werden. Bezeichnenderweise trifft dies auch auf den Notar zu, der den eigentlichen Text verfasst haben dürfte. Diese Angaben werden als unerlässliches Element der Inschrift empfunden. ${ }^{46}$ Doch sie stehen nicht allein. In Erinnerung an die Eroberung von Lucca 1314 und den Sieg über Florenz 1315 stellten die Pisaner auf der Kathedral-

zu nennen. Nicht zuletzt wegen der aktuellen bibliographischen Übersicht sei auf die Monographie von von der Höh 2006, passim und insbes. 365-370 verwiesen.

42 Vgl. Carosi 1986, 82-83 Nr. 28.

43 Vgl. Carosi 1986, 80-81 Nr. 27.

44 Zum Komplex der Inschriften aus Sardinien und insbesondere Cagliari, von denen viele von Pisa in Auftrag gegeben wurden, siehe den noch immer gültigen Aufsatz von Casini 1905.

45 Den dargestellten Fall der Inschriften aus Cagliari hat Banti 1983 erschöpfend behandelt.

46 Zur inschriftlichen Lobpreisung all jener, die an der Realisierung des architektonischen Werks beteiligt waren, äußert sich Banti 1995. Hierbei untersucht er den pisanischen Fall sowohl im spezifischen wie auch - so zeigt sich am Beispiel von Cagliari - im weiteren Sinne. 
fassade von Cagliari auch zwei Gedenkinschriften zu ebendiesen Kriegshandlungen aus. Sie verwendeten die Inschriftensprache bewusst und in völliger Überzeugung ihres unvergänglichen Wertes. Um die Worte von Ottavio Banti zu zitieren, „erstatteten sie einen regelrechten Kriegsbericht". ${ }^{47}$ Sie enthielten aber noch ein weiteres Element. Es wird nämlich präzisiert, dass zur Unterstützung der ersten Inschrift ein Stein verwendet wird, der de palatio civitatis Lucane gewonnen wurde. Auf diese Weise wird die Inschrift, die gleichzeitig faktisch eine Art Kriegstrophäe bedeutet, mit einer weiteren und impliziten politischen Botschaft aufgeladen. Diese klang auch nach einer Mahnung an die vielen inimici von Pisa.

Die Notwendigkeit, durch das geschriebene Wort nicht allein ein Ereignis, sondern auch ein wichtiges Element der städtischen Ordnung und des Alltagslebens in der Stadt zu unterstreichen, tritt anhand einzigartiger Zeugnisse hervor. Als Beispiel ist hierzu die erklärende Inschrift in der sogenannten Pietra del Vituperio von 1260 anzuführen. Der Stein wird auch als „Bankrottstein“ bezeichnet und wird aktuell im Palazzo della Ragione von Padua aufbewahrt. ${ }^{48}$

Er besteht aus einem Trachytblock, der in seiner Form einem umgekehrten kegelstumpfförmigen Sitz gleicht. Ursprünglich war der Stein im Palazzo delle Debite verortet, der früher als Gefängnis diente. Nur einem Heiligen mit dem Charisma eines Antonius von Padua konnte es im Jahr 1231 gelingen, für zahlungsunfähige Schuldner und Bankrotteure so erfolgreich einzutreten, dass die Pfahlhängen und die lebenslängliche Gefängnisstrafe abgeschafft wurden, zu denen jene zuvor verurteilt worden waren. Nun wurden sie hingegen dazu verpflichtet, sich bis auf Hemd und Unterhosen auszuziehen (hieraus leitet sich die italienische Redensart ,in Leinenhosen bleiben“ ab, die vom bildlichen in den metaphorischen Sinn „,besitzlos bleiben“ übergegangen ist). Außerdem mussten sie sich dreimal auf den Stein setzen, während sie die Worte cedo boni (,,ich verzichte auf den Besitz“) wiederholten, und daraufhin die Stadt verließen. Um die Betrachter des Steins nicht im Dunkeln zu lassen, wurde folgende Lehrschrift darauf eingraviert: Lapis vituperii et cessionis bonorum.

47 Lange wurden die beiden Inschriften für verloren gehalten, bis ein Fragment von bedeutender Größe gefunden wurde, das offenbar der zweiten Inschrift zuzuordnen ist. Es wurde auf einer Tafel identifiziert, die gegenwärtig auf der rechten Seitenwand des Doms von Cagliari eingemauert ist; vgl. Banti 1983, 273-276.

48 Vgl. http://cem.dissgea.unipd.it/schedarioriunito.pdf, Kartei Nr. 34. 


\section{Schluss}

Abschließend möchte ich versuchen, die Fäden meiner Überlegungen $\mathrm{zu}$ einem Muster zu verweben. Es hat sich herausgestellt, dass die Inschrift eine hyperkommunikative Funktion ausübt: Einerseits unterstützt sie die Schrift und somit einen Text, der erst dann einen Sinn ergibt, wenn er zum Lesen geschaffen und ausgestellt wird. Andererseits besitzt sie einen absoluten ikonischen bzw. symbolischen Wert. Hieraus ließe sich schließen, dass sich die Inschrift an Adressaten wendet, welche die inschriftliche Botschaft gemäß einer Abstufung unterschiedlicher Verständnisebenen aufnehmen können. An dieser Stelle kann ich nicht auf die Frage nach dem linguistischen Aspekt der Inschriften eingehen, die ich im Laufe meines Beitrags vernachlässigt habe. Diesbezüglich sollte ich aber zumindest anmerken, dass die inschriftliche Sprache zwischen zwei Extremen schwankt, einem hohen und einem stark vereinfachten Niveau, zwischen nüchternen, knappen Texten und ausgefeilten, sprachlich komplexen Texten, welche mit Zitaten und ausgewählten Redewendungen durchwirkt sind. Auch dürfen im Rahmen der „scritture esposte“ die Einführung und anschließende Übernahme der Vulgärsprache nicht vergessen werden, was den Leserkreis erheblich erweitert haben dürfte.

Abgesehen von der Ausführung und Organisation der Schrift und ganz unabhängig vom Lesen und Verstehen des Textes selbst, ${ }^{49}$ besitzt die Inschrift einen bildlichen Wert, da sie eine Botschaft vermittelt, in der Zeit speichert und verbreitet, die einen nahezu figurativen Sinn besitzt. Die Inschrift erinnert an ein Ereignis, fasst es zusammen und stellt es gleichsam mit Zeichen dar, die sich von den ikonographischen unterscheiden. So erweist sie sich als eine Kommunikationsform, die ebenso wirksam

49 Die graphischen Aspekte und die technische Ausführung der Inschriften, das heißt die verwendete Typologie, das Grundmodell und die handwerkliche Ausführung der Buchstaben (die in Form einer mehr oder weniger tiefen Rille eingraviert, aber auch als Relief erzeugt werden können) stellen selbstverständlich ausnahmslos Elemente dar, die sich auf das Verständnis der inschriftlichen Botschaft auswirken können. Diesbezüglich spielen auch die mise en page und die mise en texte der Inschriften, also die Art und Weise, nach der der Text auf dem Schriftgrund organisiert und skandiert wird, eine wichtige Rolle. Hierunter zählen die Weite des Zeilenabstandes sowie der Gebrauch der Interpunktions- und Zeichensetzungssysteme. Das Textverständnis gründet ferner auf die mehr oder weniger intensive Verwendung eines mehr oder weniger artikulierten Abkürzungssystems. Daneben ist selbstverständlich die Ausdruckswahl entscheidend, die - einen weiteren Aspekt der Textformulierung bildend - von der syntaktischen Organisation bis hin zur Wahl der prosaischen oder metrischen Form und schließlich der Gebrauch bestimmter Formeln reicht. Die genannten Elemente sind in jedem Fall zu berücksichtigen, um gänzlich das Publikum und die Wirksamkeit des Textes zu definieren, wenn es zutrifft, dass der materiale Aspekt des Textes dessen Verwendungsgrad bestimmt. Zusätzlich zum Text und Kontext der Inschriften stellen folglich deren materiale Struktur und Schrift Faktoren dar, die sich nicht nur mit den Funktionen der „scritture esposte“ verbinden, sondern auch miteinander interagieren und sich gegenseitig in der Bestimmung des Empfangs, der Wirksamkeit und des Erfolgs der epigraphischen Kommunikation beeinflussen. 
ist wie die durch Bilder vermittelte, mit der sie sich bisweilen verbindet, indem sie mehr oder weniger vollständig mit ihr verschmilzt.

Sollten wir aus dem, was wir beobachtet und behandelt haben, einige allgemeine Überlegungen ableiten, so könnten wir uns meines Erachtens nach darauf einigen, dass die Inschrift in der bewegten Wirklichkeit der italienischen Kommunen zweifellos als ein Kommunikations-, Verbreitungs- und Verherrlichungsinstrument diente. Zugleich war sie natürlich auch - und wahrscheinlich primär - ein Instrument zur Wahrung und Vermittlung der Erinnerung und des Gedenkens an die Geschichte der jeweiligen Stadt. Sie ist deren graphische, sicht- und lesbare Darstellung. Dies trifft umso mehr zu, wenn die Botschaft nicht nur anhand von Zeichen, sondern auch auf eine integrierte und vollständige Weise durch die Entfaltung und Realisierung eines mehr oder weniger artikulierten ikonographischen Programms ausgedrückt wird. ${ }^{\mathbf{5 0}}$ Die Inschriften sind nicht ausschließlich oder vorrangig ein wirksames Instrument der Verwaltungstätigkeit sowie der politischen Entscheidungen. Sie bekräftigen gewissermaßen auch die Verbindungen zwischen den führenden Schichten und den Stadtbürgern, die in den Handlungen der ersten gegenüber den zweiten begründet ist. Zudem können die Inschriften zum konkreten wie ideellen „Ort der Erinnerung“ werden. Dies gilt sowohl für denjenigen, der die Inschriften schuf, sie a priori als ein documentum verfasste und als solches las, als auch für jene, die sie a posteriori als ein eigentliches monumentum interpretieren, wie wir es gegenwärtig beim Lesen tun. Als Beispiel mag an dieser Stelle die Komplexität der Fontana Maggiore von Perugia in Erinnerung gerufen werden, in der die Schrift fortwährend vom strukturellen zum überstrukturellen Element mutiert. Sie zeigt uns, wie der epigraphische Text, der an einen bestimmten historischen Zeitpunkt, genauer an ein bestimmtes Ereignis gebunden ist, im kommunalen Mittelalter eine besondere Bedeutung erlangte. Der Text ordnet das Werk, mit dem es zusammenhängt, in das Leben, die Geschichte und Erinnerung der Stadtgemeinschaft ein, indem es die Narration der Gleichzeitigkeit zeitlich verschiebt und wiederholt.

Doch nicht nur das. Wir sind stets dazu aufgerufen, uns darüber bewusst zu werden, dass die Inschriftenkultur (im vorliegenden Fall die kommunale Inschriftenkultur) ein mächtiges Kommunikationssystem ist, welches das Potential der Schrift verstärkt. Indem es ideell und erfolgreich die flache Linearität der Schriftfolge überwindet und vom Gedenken zur Propaganda übergeht, ist es zusätzlich ein Instrument, das individuelle und kollektive Verhaltensweisen zu beeinflussen vermag. Entsprechend kann die Epigraphik die öffentliche Meinung lenken und Betrachtungsweisen durchsetzen, die als Interpretationsmodelle der Gegenwart, aber auch vergangener Wirklichkeiten angenommen werden. So schlägt dies Vincent Debiais in

$50 \mathrm{Zu}$ präzisieren ist zusätzlich: „[L]e iscrizioni apposte ai palazzi di nuova costruzione o incise sulle fontane e acquedotti sono così l'espressione del raggiungimento di una pacificazione interna e di una stabilità delle istituzioni.“ Hierin teile ich die Ansicht von Bottazzi 2006, 357. 
einem ausführlichen und eindrucksvollen Aufsatz über die Erzeugung und Verbreitung von Erinnerung innerhalb der Inschriften vor. ${ }^{51}$ Die Inschriften erweisen sich in diesem Sinne als ein wertvolles Instrument der Propaganda, indem sie alle Räume des mittelalterlichen Lebens besetzen und sich im Sicht- und Denkhorizont der cives behaupten.

\section{Literaturverzeichnis}

Augenti, Andrea/Munzi, Massimiliano (1997), Scrivere la città. Le epigrafi tardoantiche e medievali di Volterra (secoli IV-XIV), Florenz.

Avarucci, Giuseppe (1978), L'iscrizione del 1230 della Porta di Solestà di Ascoli Piceno, Ascoli Piceno. Banti, Ottavio (1983), „Epigrafi e propaganda politica ai primi del Trecento. Note di epigrafia e di storia medioevale“, in: Quaderni ticinesi di numismatica e antichità classiche 12, 257-276.

Banti, Ottavio (1992), „Epigrafi ,documentarie‘, ,chartae lapidariae“ e documenti (in senso proprio). Note di epigrafia e di diplomatica medievali“, in: Studi medievali 33, 229-242.

Banti, Ottavio (1995), „Operai, architetti e attività edilizia del Comune di Pisa nelle epigrafi tra il XIII e il XIV secolo“, in: Silio Pietro Paolo Scalfati (Hg.), Ottavio Banti, Scritti di storia, diplomatica ed epigrafia, Ospedaletto (Pisa), 159-180.

Banti, Ottavio (2000), Monumenta epigraphica pisana saeculi XV antiquiora, Pisa.

Bartoli Langeli, Attilio (1983), Codice diplomatico del Comune di Perugia. Periodo consolare e podestarile (1139-1254), Bd. 1: 1139-1237, Perugia.

Bartoli Langeli, Attilio/Giovè Marchioli, Nicoletta (1996), „Le scritte incise della Fontana Maggiore“, in: Carlo Santini (Hg.), Il linguaggio figurativo della Fontana Maggiore di Perugia, Perugia, 163-195.

Bartoli Langeli, Attilio/Merli, Sonia (2013), „Un aspetto della committenza pubblica in ambito urbano: le fontane“, in: Silvia Maddalo u. Isa Lori Sanfilippo (Hgg.), Civiltà urbana e committenze artistiche al tempo del Maestro di Offida (secoli XIV-XV) (Tagung Ascoli Piceno 2011), Rom, 69-102.

Bartoli Langeli, Attilio/Zurli, Loriano (1996), L'iscrizione in versi della Fontana maggiore di Perugia (1278), Rom.

Benucci, Franco (Hg.) (2015), Corpus dell'Epigrafia Medievale di Padova, Bd. 1: Le iscrizioni medievali dei Musei Civici di Padova. Museo d'Arte medievale e moderna, Sommacampagna.

Benucci, Franco/Foladore, Giulia (2008), ,,Iscrizioni parlanti“ e ,iscrizioni interpellanti“ nell'epigrafia medievale padovana, in: Padua Working Papers in Linguistics 2, 56-133, online zugänglich unter: http://www.disll.unipd.it/padua-working-papers-linguistics (zuletzt aufgerufen am 10.11.2018).

Bottazzi, Marialuisa (2006), „Tra Papato e Impero. L'uso dell'epigrafia nei secoli XI e XII a Viterbo“, in: Studi medievali 47, 305-360.

Bottazzi, Marialuisa (2012a), „Città e scrittura epigrafica“, in: Miriam Davide (Hg.), Identità cittadine e aggregazioni sociali in Italia, secoli XI-XV (Tagung Triest 2010), Triest, 275-290.

51 Siehe zu diesem Aspekt Debiais 2007. Er hat erneut und extensiver die öffentlich ausgestellten Schriften, die einen polyvalenten Bestandteil der Alltagslandschaft bildeten, als ein unabdingbares Element der spätmittelalterlichen Städte identifiziert, siehe Debiais 2009. 
Bottazzi, Marialuisa (2012b), Italia medievale epigrafica. L'alto medioevo attraverso le scritture incise (secc. $I X-X I)$, Triest.

Bottazzi, Marialuisa (2015), „La porta Romana (1171). Un luogo della memoria e della distruzione della città“, in: Pietro Silanos u. Kai-Michael Sprenger (Hgg.), La distruzione di Milano (1162). Un luogo di memorie, Mailand, 55-83.

Breveglieri, Bruno (1989), „La scrittura epigrafica in età comunale: il caso bolognese“, in: Civiltà Comunale: Libro, Scrittura, Documento (Tagung Genua 1988; Atti della Società Ligure di Storia Patria n. s. 29,2), Genua, 387-432.

Bussolati, Cristina (1988), „Per i luoghi, le funzioni e le forme delle croci in pietra del Museo civico medievale“, in: /l carrobbio 14, 75-84.

Carosi, Attilio (1986), Le epigrafi medievali di Viterbo (secc. VI-XV), Viterbo.

Casini, Tommaso (1905), „Le iscrizioni sarde del medioevo“, in: Archivio storico sardo 1, 303-380.

De Rubeis, Flavia (2016), „La gotica epigrafica in Italia tra sud e nord. Tra manoscritti ed epigrafi, tra sculture e miniature“, in: Scripta 9, 57-71.

Debiais, Vincent (2007), „Afficher pour convaincre: la construction et la promotion de la mémoire dans les inscriptions comme instrument de la propagande médiévale“, in: Martin Aurell (Hg.), Convaincre et persuader: communication et propagande aux XIIe et XIIIe siècles (Tagungen Fontevrault 2004, Oxford 2005, Barcelona 2006 u. Saintes 2006), Poitiers, 649-702, online zugänglich unter: https://halshs.archives-ouvertes.fr/halshs-00343562 (zuletzt aufgerufen am 10.11.2018).

Debiais, Vincent (2009), Messages de pierre. La lecture des inscriptions dans la communication médiévale (XIIIe-XIVe siècle), Turnhout.

Dufour Bozzo, Colette (1989), La porta urbana nel medioevo. Porta Soprana di Sant'Andrea in Genova, immagine di una città, Rom.

Favreau, Robert (1991), „Le thème iconographique de la porte“, in: Cahiers de civilisation médiévale 34, 267-279.

Franceschini, Adriano (1969), I frammenti epigrafici degli statuti di Ferrara del 1173 venuti in luce nella cattedrale, Ferrara.

Franceschini, Adriano (1972), „Nuovi frammenti epigrafici degli statuti di Ferrara del 1173“, in: Atti e memorie della Deputazione provinciale ferrarese di Storia patria 11 (3. ser.), 101-108.

Gardner, Julian (1987), „An Introduction to the Iconography of the Medieval Italian City Gate“, in: Dumbarton Oaks Papers 41, 199-213.

Giovè Marchioli, Nicoletta (1994), „L'epigrafia comunale cittadina“, in: Paolo Cammarosano (Hg.), Le forme della propaganda politica nel Due e nel Trecento (Tagung Triest 1993; Collection de l'École Française de Rome 201), Rom, 263-286.

Gouédo-Thomas, Catherine (1992), „Les fontaines médiévales. Images et réalité“, in: Mélanges de l'École Française de Rome - Moyen Âge 104, 507-517.

Gozzadini, Giovanni (1863), „Delle croci monumentali ch'erano nelle vie di Bologna nel secolo XIII“, in: Atti e memorie della Deputazione di Storia Patria per le province di Romagna 2, 27-69.

Gramigni, Tommaso (2012), Iscrizioni medievali nel territorio fiorentino fino al XIII secolo, Florenz.

Grandi, Renzo (1978/79), „Le tombe dei dottori bolognesi: ideologia e cultura“, in: Atti e Memorie della Deputazione di storia patria per le province di Romagna n. s. 29/30, 163-181.

Grandi, Renzo (1982), I monumenti dei dottori e la scultura a Bologna (1267-1348), Bologna.

Guerrini, Paola (2013), „Scrivere sulle mura. Le iscrizioni dei sistemi difensivi nella penisola italiana tra VI e XIII secolo: note preliminari“, in: Temporis signa 8, 1-37.

von der Höh, Marc (2006), Erinnerungskultur und frühe Kommune. Formen und Funktionen des Umgangs mit der Vergangenheit im hochmittelalterlichen Pisa (1050-1150), Berlin.

I litorali toscani e la Maremma (1982), Mailand. 
La costruzione della città comunale italiana (secoli XII-inizio XIV) (Tagung Pistoia 2007), Pistoia 2009.

Licandro, Orazio (2002), Il ,diritto inciso: Lineamenti di epigrafia giuridica romana, Catania.

Müller, Rebecca (2002), Sic hostes lanua frangit. Spolien und Trophäen im Mittelalterlichen Genua, Weimar.

Origone, Sandra/Varaldo, Carlo (Hgg.) (1983), Corpus Inscriptionum Medii Aevi Liguriae, Bd. 2: Genova. Museo di S. Agostino, Genua.

Panero, Francesco/Pinto, Giuliano (Hgg.) (2009), Castelli e fortezze nelle città e nei centri minori italiani (secoli XIII-XV) (Tagung Cherasco 2008), Cherasco.

Pantarotto, Martina (2012), „Il giglio e la croce sulle mura di Firenze“, in: Opera Nomina Historiae 7, 67-88, online zugänglich unter: http://onh.giornale.sns.it (zuletzt aufgerufen am 10.11.2018).

Petrucci, Armando (1985), „Potere, spazi urbani, scritture esposte: proposte ed esempi“, in: Culture et idéologie dans la genèse de l'État moderne (Tagung Rom 1984; Collection de l'École Française de Rome 82), Rom, 85-97.

Petrucci, Armando (1986), La scrittura. Ideologia e rappresentazione, Turin.

Pini, Ivan (1999), Città, chiesa e culti civici in Bologna medievale, Bologna.

Rolandino (2004), Vita e morte di Ezzelino da Romano. Cronaca, hg. von Flavio Fiorese, Mailand.

Salvi, Antonio (1999), Iscrizioni medievali di Ascoli, Ascoli.

Scalia, Giuseppe (1963), „Epigraphica pisana. Testi latini sulla spedizione contro le Baleari del 1113-1115 e su altre imprese anti-saracene del secolo XI. L'iscrizione per le imprese di Reggio Calabria, della Sardegna e di Bona“, in: Miscellanea di studi ispanici 6, 234-286.

Scalia, Giuseppe (1972), „,Romanitas“ pisana tra XI e XII secolo. Le iscrizioni romane del duomo e la statua del console Rodolfo“, in: Studi Medievali 13, 791-843.

Schmid, Wolfgang (1998), „Brunnen und Gemeinschaft im Mittelalter“, in: Historische Zeitschrift 267, 561-586.

Schulze, Ulrich (1994), Brunnen im Mittelalter. Politische Ikonographie der Kommunen in Italien, Frankfurt a. M.

Silva, Augusta (Hg.) (1987), Corpus Inscriptionum Medii Aevi Liguriae, Bd. 3: Genova. Centro storico, Genua.

Ulianich, Boris/Vitolo, Giovanni (Hgg.) (2001), Castelli e cinte murarie nell'età di Federico II (Tagung Montefalco 1994), Rom.

Visentin, Laura (2016), Alle fonti del Medio Evo. Fontane medievali dal XIII al XIV secolo in Italia, Arezzo. 